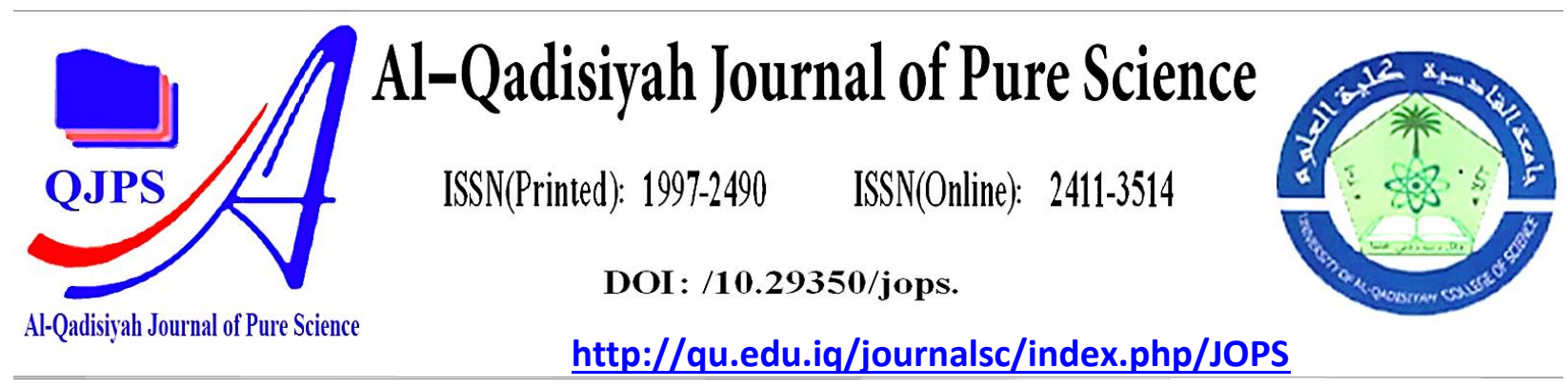

\title{
Synthesis, identification and evaluation of antibacterial activity for some new heterocyclic derivatives from 4-methoxy-2-nitroaniline
}

\begin{tabular}{|c|c|}
\hline $\begin{array}{l}\text { Authors Names } \\
\text { a.Sabah Matrood Mezaal } \\
\text { b. Shaimaa Adnan }\end{array}$ & ABSTRACT \\
\hline b. Shaimaa Adnan & $\begin{array}{l}\text { This research involved synthesis. novel heterocyclic derivatives } \\
\text { (quinazoline and thiazinone) derivatives, this compounds prepared from }\end{array}$ \\
\hline Article History & starting react (4-methoxy-2-nitroaniline) with \\
\hline $\begin{array}{l}\text { Received on: } 21 / 6 / 2021 \\
\text { Revised on: } 10 / 7 / 2021 \\
\text { Accepted on: } 11 / 7 / 2021\end{array}$ & $\begin{array}{l}\text { dimethoxyacetophenone to gate azo derivative }(A),(A) \text { interact with } \\
\text { aromatic amine derivatives to produce imine compounds }(B 1-B 2) \text {, imine }\end{array}$ \\
\hline $\begin{array}{l}\text { Keywords: } \\
\text { schiff base, Oxazepine, } \\
\text { Quinazoline , Thiazine }\end{array}$ & $\begin{array}{l}\text { derivatives interact with (anthranilic acid , 2-mercaptobenzoic) to get } \\
\text { heterocyclic derivatives quinazoline (C1-C2) and thiazinone (D1-D2). All } \\
\text { these compound characterized by13 C-NMR. FT-IR, 1HMNR. Then }\end{array}$ \\
\hline $\begin{array}{l}\text { DOI: https://doi.org/10 } \\
\text { jops.2021.26. } 4.1383\end{array}$ & $\begin{array}{l}\text { that,we studies the, biological,properties for,all heterocyclic } \\
\text { derivatives,to, ward.two different kindof bacteria. }\end{array}$ \\
\hline
\end{tabular}

\section{Introduction}

Heterocyclic,compounds that, have $(\mathrm{N}, \mathrm{S})$ as hetero atoms, are very, important because, of, the applications ${ }^{(1)}$ Heterocyclic compounds forms a part of large number,of pharmaceutical relevant molecule andhave major biological significance ${ }^{(2)}$ Organic heterocyclic compounds currently account for about $70 \%$ of all clinically used drugs ${ }^{(3)}$ Azo,compounds,or.dyes are,characterized, by, the,presenc of the,Azo,moiety $(-\mathrm{N}=\mathrm{N}-)$ ).)in their,structure,conjugated with two,distinct or,identical,,monoor,poly-cyclic.aromatic.or heteroaromatic. systems ${ }^{(4)}$. Thebiological,importance of,Azo,compounds is, well,known due to their use as inflammatory ${ }^{(5)}$, antibacterial ${ }^{(6)}$, anti-diabetic ${ }^{(7)}$,and antifungal ${ }^{(8)}$. Schiff bases are condensation products of primary amines and carbonyl compounds and they were discovered by Hugo Schiff in $1864^{\cdot(9)}$. A,Schiff base, is the nitrogen, analogue of,aldehyde in, which the

\footnotetext{
a Department of chemistry, College of Education for, Al-Qadisiyah University, Sabah Matrood, Iraq, E-Mail: sabahmatrod1968@gmail.com

b Department of chemistry, College of Education for, Al-Qadisiyah University, ${ }^{1}$ Shaimaa Adnan, Iraq, E-Mail: shaimaa.adnan@qu.edu.iq, shemaadnan@yahoo.com
} 
$\mathrm{C}=\mathrm{O}$ group,is replaced, by $\mathrm{a}, \mathrm{C}=\mathrm{N}$ group ${ }^{(10)}$.There are numbers, of, biologically. important,Schiff, bases have, been noted in,previous,study possessin,, antibacterial, antifungal,antimicrobial,,anticonvulsant.,antitumor.,,anti-inflammatory, and, anti,HIV,activities ${ }^{(11) .}$

Quinazolines,and,thiazine,derivatives, have,six-.membered,containing,( $N$,and,S), respectively such as antitubercular, anti-inflammatory, antimicrobial , antipyretic. , anti-HIV , analgesic , antitumor , and calcium channel modulatory activities ${ }^{(12),}$ thiazine derivatives, that exhibit,various, biological, activities, such, as anti-tubercular,, antifungal $^{(13)}$ insecticidal,and, pesticidal $^{(14)}$,Studies, of, heterocycles,containing, hetero, atoms, such as Sulphur and Nitrogen,,are definitel,one of,the supreme,targeted areas, in heterocyclic,chemistry. They, are extensively, used in, several studies, of natural,products, and pharmaceutical,agent's, synthesis Thiazine ring,systems are,considered a,significant,heterosystem in, heterocyclic,chemistry ${ }^{(15)}$, Quinazoline nucleus,is an,interesting molecule,among the,most important,classes of,an aromatic,bicyclic,compounds with two,nitrogen atoms in,their structure, It is, consisting of,aromatic, benzopyrimidine,system,Synthesis ${ }^{(16)}$ made up,of two,fused six member simple,aromatic rings,benzene,and pyrimidine, ring $^{(17) \text {, }}$, Researchers, have already, determined,many, therapeutic,activities, of,quinazoline,derivatives,,including,,nticancer ${ }^{(18),}$ antiviral $\left.^{(19)}\right)$ "antiviral 'antimalarial ${ }^{(20)}$, ,.

\section{Materials}

"(FTIR.),Spectra,(400., $\quad-4000 \quad$.cm-1), in $\quad \mathrm{KBr}$,,disk, were, recorded,on,SHIMADZU.FTIR,8400S,Fourier transform. ${ }^{13} \mathrm{C}-\mathrm{NMR}$, and ${ }^{1} \mathrm{HNMR}$ were recorded on varian agilant USA at $(500 . \mathrm{MHz})$ with.(DMSO.-d6) measure.ement were.made at Department ofChemistry,Tehran University,,Iran."

\subsection{Preparation of compound $A^{(21)}$}

(0.01) (1.68 g) of 4-methoxy-2-nitro aniline, was dissolved in a solution consisting of (10 $\mathrm{ml}$ ), hydrochloric acid with the mixture cooled,to. $(0,-5){ }^{\circ} \mathrm{C}$ and..then added sodiumnitrite $(0.8 \mathrm{~g})$ $\mathrm{NaNO}_{2}$ with a brown color drop as a drop to a solution consisting of $(1.8 \mathrm{~g})(0.01 \mathrm{~mol})$ of 2,4dimethoxyacetophenone and $(2 \mathrm{~g})$ of $\mathrm{NaOH}$ dissolved in $(130 \mathrm{ml})$ distilled and cooled water to $\left(20^{\circ} \mathrm{C}\right)$ and $(10 \mathrm{ml})$ ethanol was,observed The Azo composite deposit is dark brown color after completing the addition process. This process was carried out in $\mathrm{PH}=5$ and the solution is left for ,(24 hours) after which the precipitate was filtered and then the precipitate was collected after filtering and washed with distilled water, and dried and recrystallized with ethanol.

\subsection{Preparation of compound (B1-B2)(22)-}

In a double-beaker flask,(1g) $(0.00278 \mathrm{~mol})$ of derivative(A) was mixed with(0.303g) $(0.00278 \mathrm{~mol})$ of $\quad$-amino phenol, $(0.303 \mathrm{~g})$ of,2-amino phenol, compound with (20ml) of ethyl alcohol added to it (three drops) of,glacial.acetic.acid and the,.mixture up .and ,left For a period of two hours, at a,temperature , $(78 \mathrm{oC})$ and then cool the mixture and leave it for ( 24 hours) and then re-crystallize it with absolute ethyl alcohol. 


\subsection{Preparation of compound (C1-C2):-}

$(0.00150 \mathrm{~mol})(0.7 \mathrm{~g})$ of dissolved schiff base B1and B2 compound was mixed in (1-4-dioxane $(20 \mathrm{ml})$ with $(0.213 \mathrm{~g})$ of (anthranilic acid) or $(0.00150 \mathrm{~mol})(0.7 \mathrm{~g})$ of Schiff base B2, was mixed in (1-4dioxane $(20 \mathrm{ml})$ with $(0.205 \mathrm{~g})$ of (anthranilic acid) was mixed in (1-4-dioxane $(20 \mathrm{ml})$ with $(0.213 \mathrm{~g})$ of (anthranilic acid) then add drops of (DMF) and reflex of (36 Hour) and then re-crystallized the product with absolute ethanol.

\subsection{Preparation of compound (D1-D2):-}

$(0.7 \mathrm{~g})(0.00155 \mathrm{~mol})$ of Schiff baseB1,B2 compound was mixed in $(22 \mathrm{ml})$ of benzene with $(0.239 \mathrm{~g})$ of 2-mercapto benzoic acid $(3 \mathrm{ml})$ of DMF then add drops of triethylamine to the reaction mixture and from Then reflex of (10 hours), then the product was filtered and re-crystallized with absolute ethanol . 


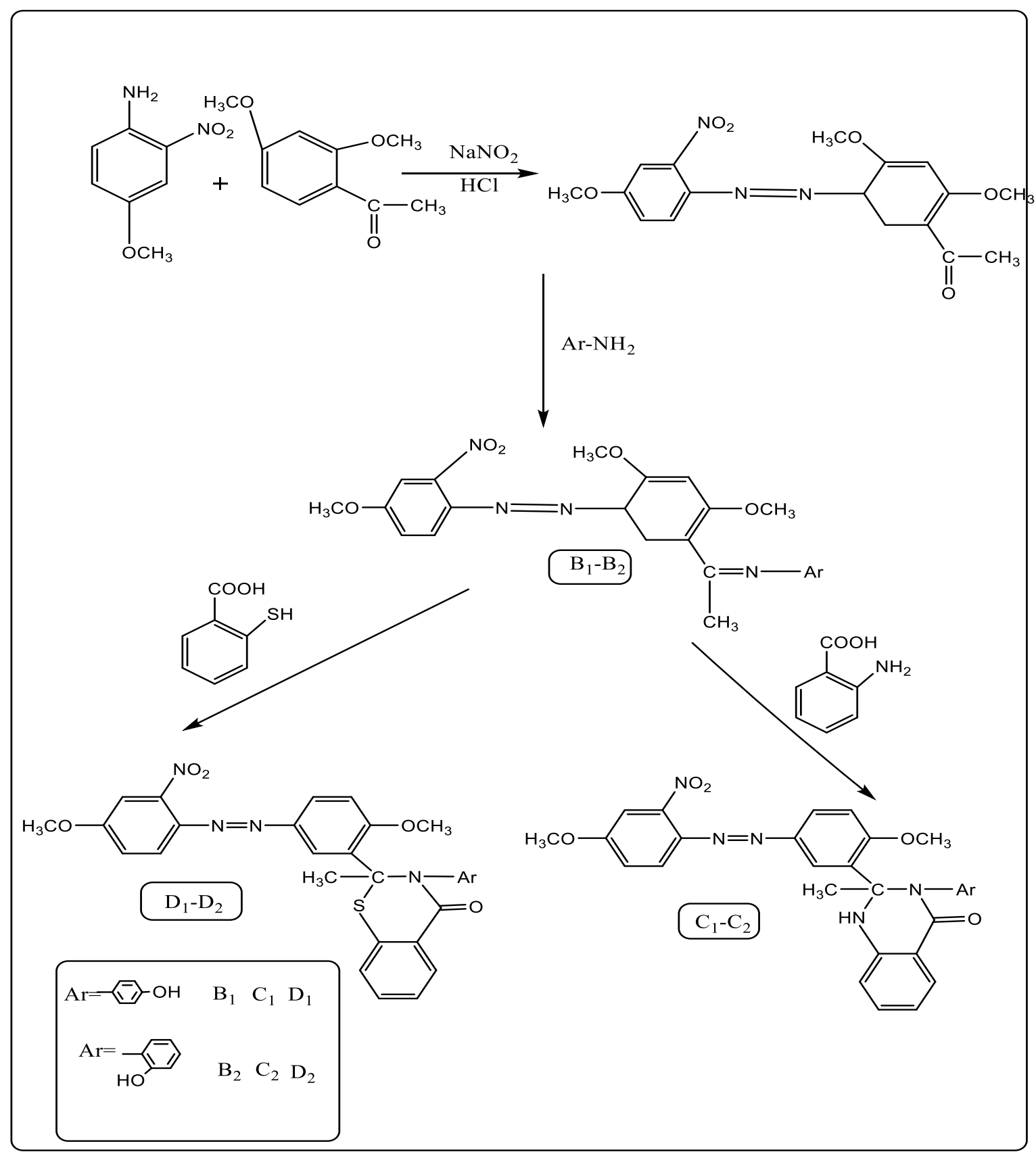

Scheme(1,) prepare ofsome, .heterocyclic, compounds

\section{Results and Discussion}

\section{Derivative (A)}

\section{(E)-1-(2,6-dimethoxy-3-((E)-(4-methoxy-2-nitrophenyl)diazenyl)phenyl)-N-(4-nitrophenyl)ethan-1-} imine $\mathbf{C 4}, \mathrm{C}$

FT-.IR,spectrum,data for,.derivative (A), show.peak at 3000 for $(\mathrm{Ar}-\mathrm{H}), 2980 \mathrm{~cm}^{-1}$ for(,C- H) in $\mathrm{CH}_{3}, 1700 \mathrm{~cm}^{-1}$ for $(\mathrm{C}=\mathrm{O}), 1650 \mathrm{~cm}^{-1}$ for , $(\mathrm{C}=\mathrm{C}),(1521-1357) \mathrm{cm}^{-1}$ for $\left(\mathrm{NO}_{2}\right) .{ }^{1} \mathrm{HMNR}$ spectrum data of derivative (A) show 2.52ppm (DMSO), 3.78ppm (S ,3H, $\left.\left(\mathrm{OCH}_{3}\right)_{13}\right), 3.87 \mathrm{ppm}\left(\mathrm{S}, 3 \mathrm{H},\left(\mathrm{OCH}_{3}\right)_{14}\right)$, 3.98ppm (S ,3H, $\left.\left(\mathrm{OCH}_{3}\right)_{15}\right)$, 3.9ppm (s, $\left.1 \mathrm{H}, \mathrm{CH}_{3}\right), 6.3-7.6 p p m(\mathrm{M}, 5 \mathrm{H}, \mathrm{Ar}-\mathrm{H})$, 9.4PPm.The C13-NMR 
spectrum data ( DMSO) compound (A) show :197ppm $\left(C_{17}\right), 79 p p m\left(C_{16}\right)$ 56ppm $\left(C_{15}\right), 55 p p m\left(C_{14}\right)$, $31 p p m\left(C_{13}\right), 164 p p m\left(C_{3}\right), 161 p p m\left(C_{4}, C_{7}\right), 98-132 p p m$ (CArom)).

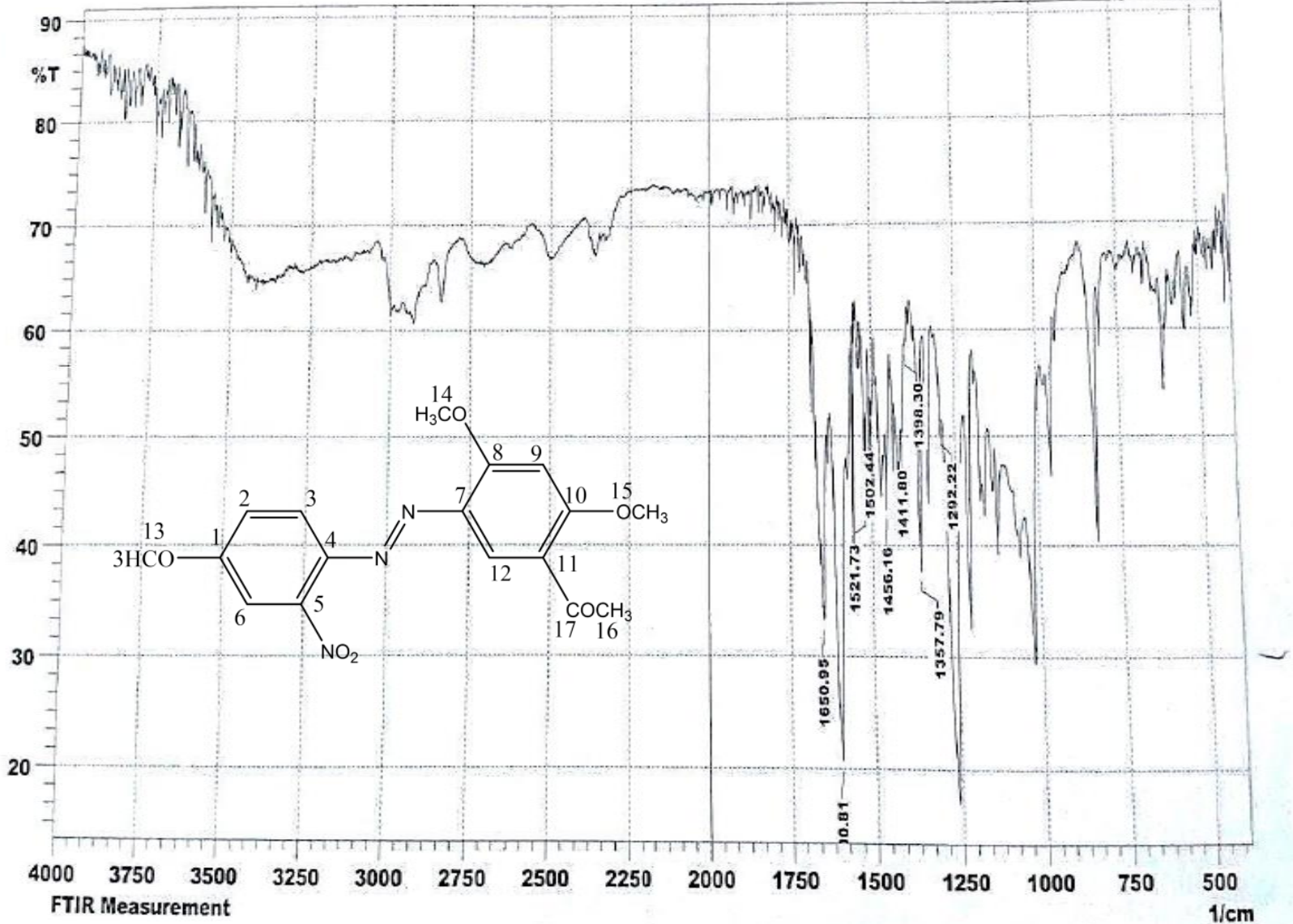

Fig(1) FTIR spectrum of compound(A) 


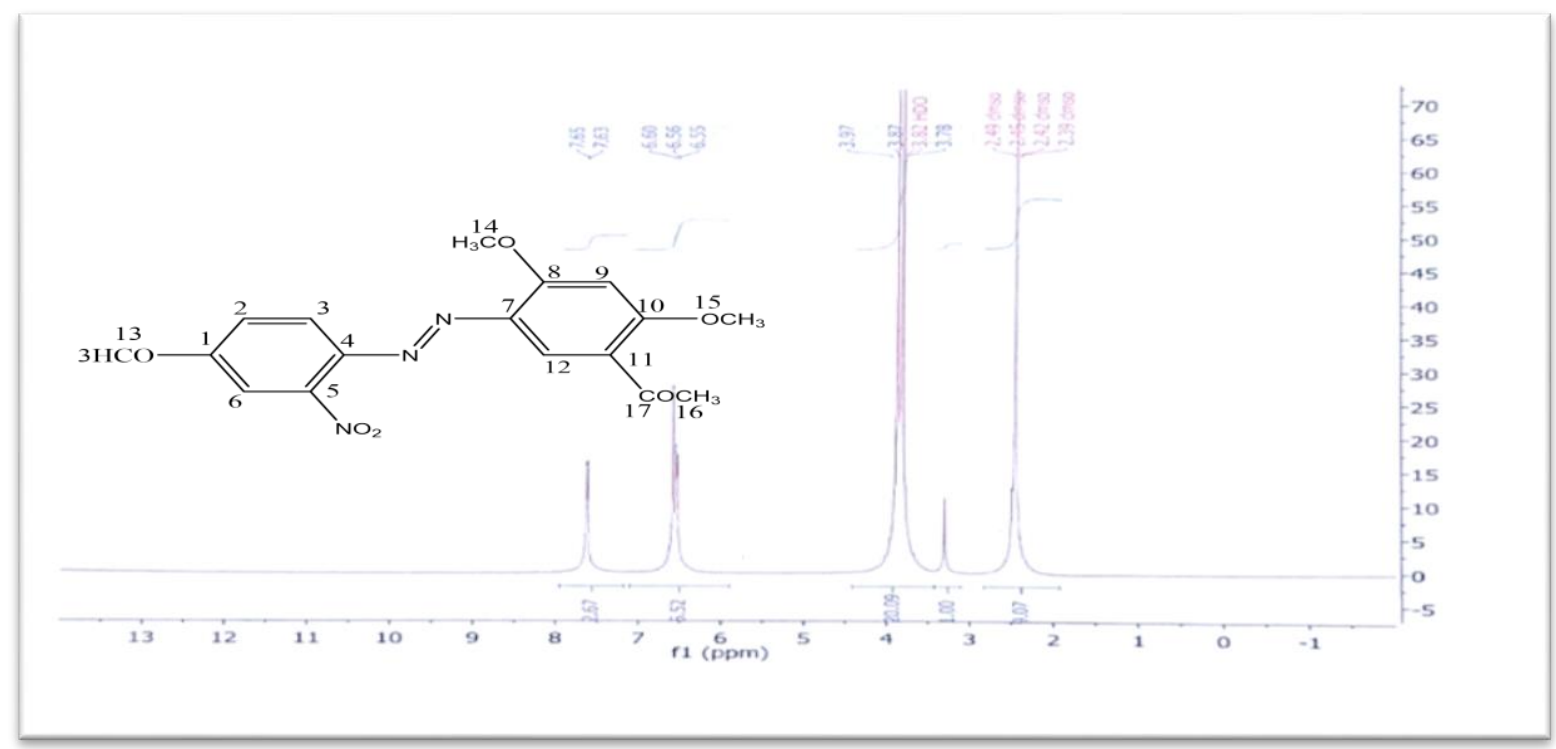

Fig.(2) $\left({ }^{1}\right.$ HNMR), spectrum, of.compound(A)

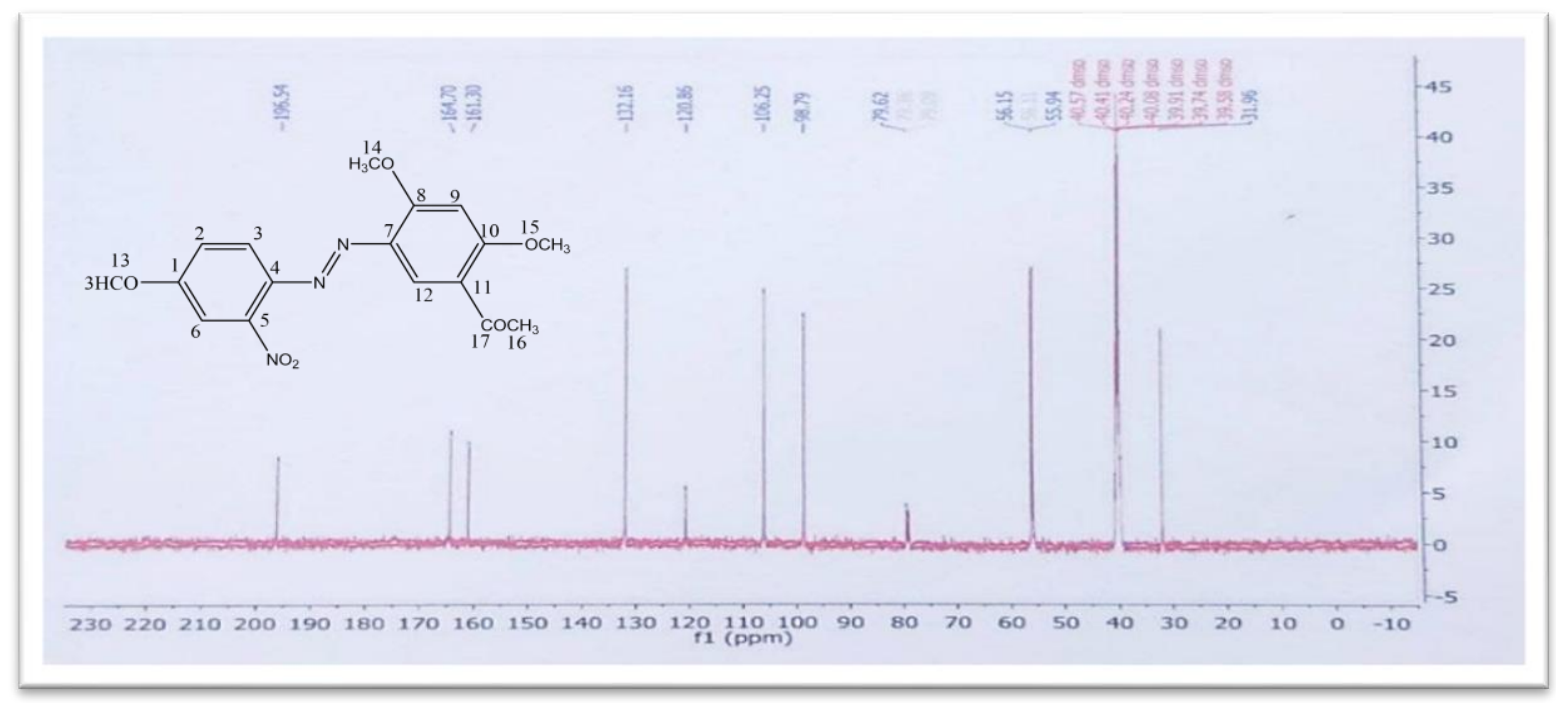

Fig.(3) (13CNMR)spectrum of,compound(A)

\section{Derivative (B1) 4-(((Z)-1-(2,4-dimethoxy-5-((E)-(4-methoxy-2-nitrophenyl)diazenyl)phenyl)} ethylidene) amino)phenol

FT-IR spectrum data for derivative (B1) show peak at, 3025 for $(\mathrm{Ar}-\mathrm{H}), 2988 \mathrm{~cm}^{-1}$ for $(\mathrm{C}-\mathrm{H})$ in $\mathrm{CH}_{3}, 1456$ forN=N, $1652 \mathrm{~cm}^{-1}$ for $\mathrm{C}=\mathrm{N}, 1500,1350 \mathrm{~cm}^{-1}$, for. $\mathrm{NO}_{2}, 1650 \mathrm{Cm}^{-1}\left(\mathrm{C}=\mathrm{C}\right.$ )arom . ${ }^{1} \mathrm{HMNR}$ spectrum data of derivative (B1) show $2.52 \mathrm{ppm}$ (DMSO) , $2.9\left(\mathrm{~S}, .3 \mathrm{H},\left(\mathrm{OCH}_{3}\right)_{19}\right), 3.9\left(\mathrm{~S},, 3 \mathrm{H}_{,},\left(\mathrm{OCH}_{3} .\right)_{21}\right)$ ,3.7 (S ,3H, $\left.\left(\mathrm{OCH}_{3}\right)_{22}\right)$, 3.8ppm $(\mathrm{s}, 1 \mathrm{H}, \mathrm{CH}),(, 6.5-7.6) \mathrm{ppm}(\mathrm{m}, 9 \mathrm{H},,(\mathrm{Ar}-\mathrm{H}), .$. , The C13-NMR spectrum data ( DMSO) compound,(B1), show :79ppm $\left(C_{21}\right), 55 p p m\left(C_{20}\right), 79 p p m\left(C_{21}\right), 31 p p m\left(C_{22}\right), 161 p p m$ $\left(C_{13}\right), 164 p p m\left(C_{4}, C_{7}\right), 196 p p m\left(C_{17}\right), 34$ ppm $\left(C_{19}\right)$. 


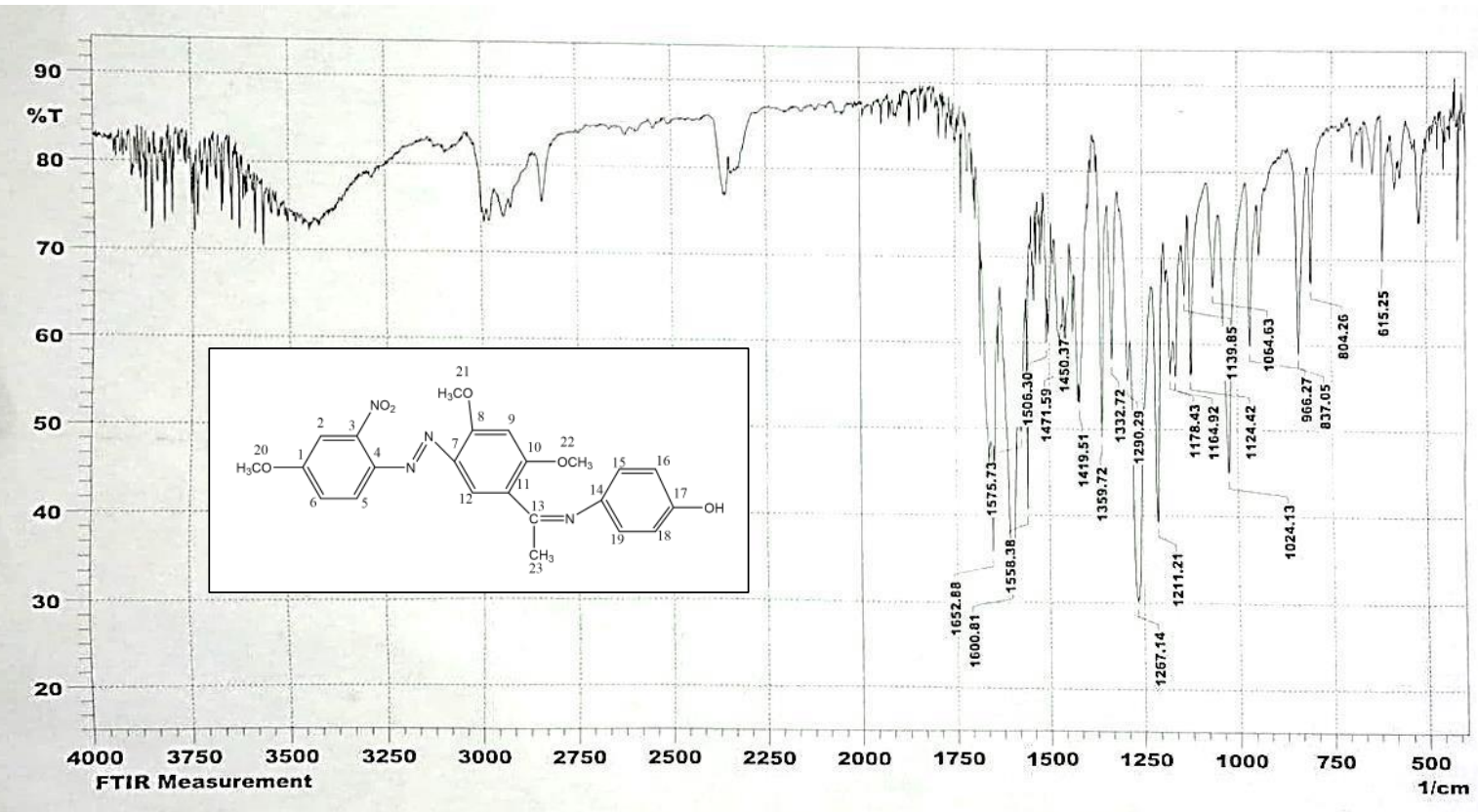

Fig(4) FT-IR,spectrum of,compound,(B1)

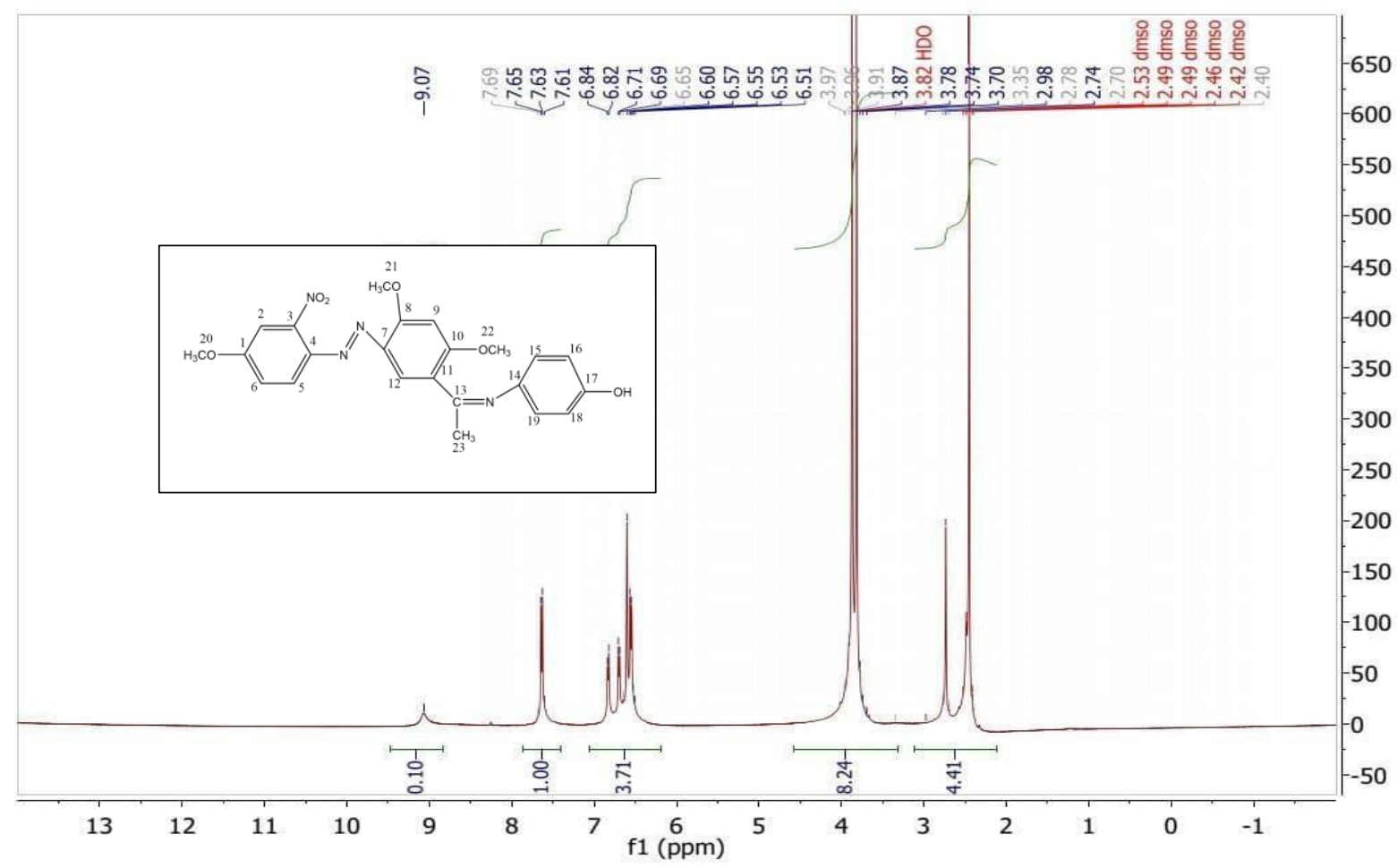

Fig(5). $\left({ }^{1}, \mathrm{H}-\mathrm{NMR},\right)$ spectrum, of,compound,(B1) 


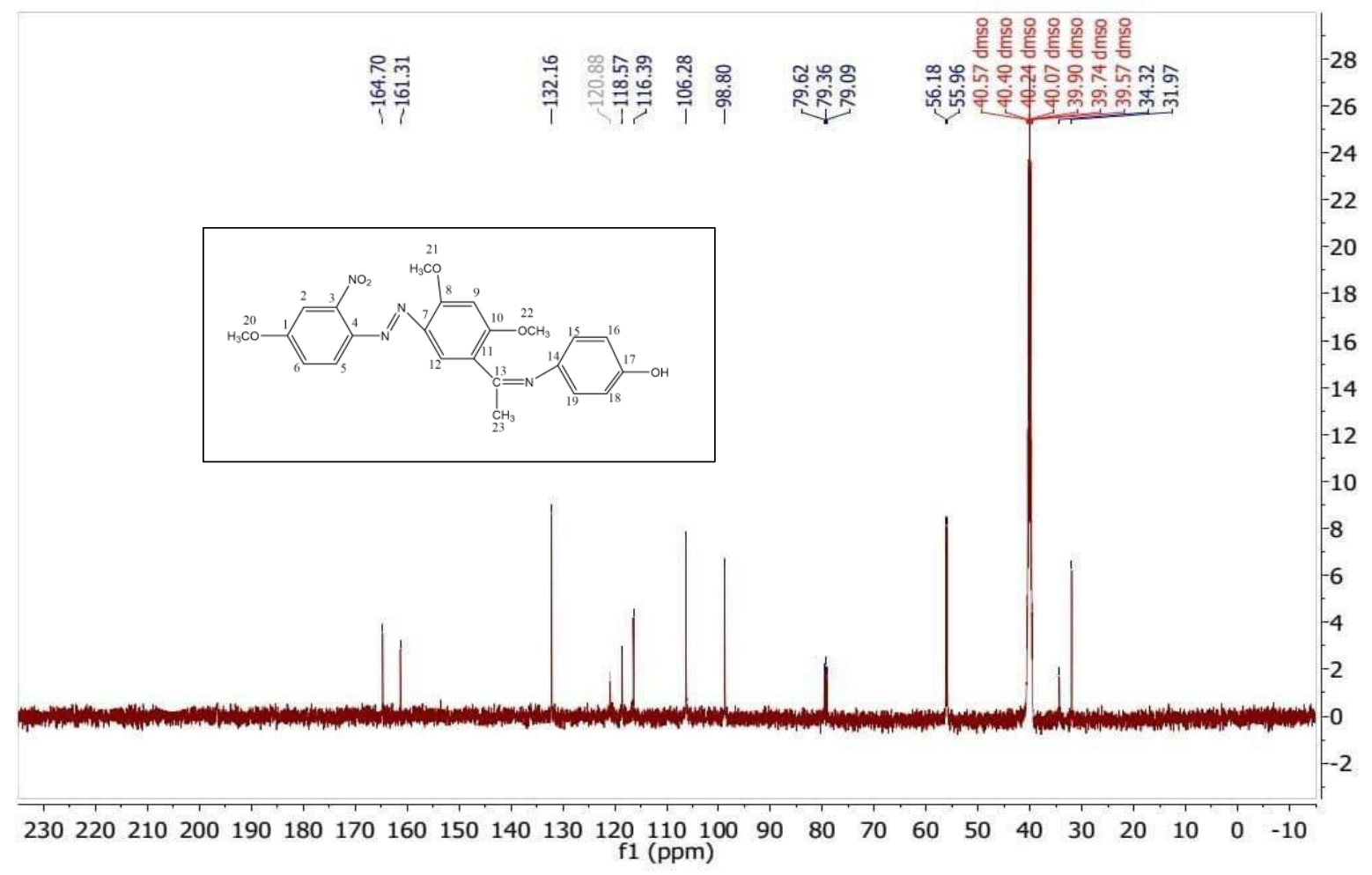

Fig.(6) $\left({ }^{13} \mathrm{C},-\mathrm{NMR}\right)$. spectrum, of compound.(B1)

\section{Compound,(B2) \\ 2-(((Z)-1-(2,4-dimethoxy-5-((E)-(4-methoxy-2-nitrophenyl)diazenylphenyl) ethylidene)amino)phenol}

FT-IR,spectrum, data for, derivative,(B2) show, band, at $3300 \mathrm{~cm}^{-1,}$ for $(\mathrm{O}-\mathrm{H}) 3080$ for $(\mathrm{Ar}-\mathrm{H})$, $2985 \mathrm{~cm}^{-1}$ for $(\mathrm{C}-\mathrm{H})$ inCH $\mathrm{CH}_{3}, 1600 \mathrm{~cm}^{-1}$ or $(\mathrm{C}=\mathrm{N}), 1593 \mathrm{~cm}^{-1}$, for . $(\mathrm{C}=\mathrm{C}), 1470 \mathrm{~cm}^{-1}$ forN=N, ${ }^{1} \mathrm{HMNR}$ spectrum data of derivative (B2) show 2.52ppm (DMSO) , $1.9\left(\mathrm{~S}, 3 \mathrm{H},\left(\mathrm{OCH}_{3}\right)_{22}\right), 3.9\left(\mathrm{~S}, 3 \mathrm{H}, .\left(\mathrm{CH}_{3}\right)_{21}\right), 3.8(\mathrm{~S}, 3 \mathrm{H}$, $\left(\mathrm{OCH}_{3}\right)_{20}, 3.7,\left(\mathrm{~S} .,, 3 \mathrm{H},\left(, \mathrm{OCH}_{3}\right)_{19}\right), 2.01\left(\mathrm{~S}, 3 \mathrm{H}, .,\left(\mathrm{OCH}_{3}\right)_{18}\right), 9.1(\mathrm{~S}, 1 \mathrm{H}(\mathrm{OH}), 6.5-7.6 p p m(m, 9 H, ~ A r-H)$. The C13-NMR spectrum data ( ,DMSO),compound (B1) show :79ppm $\left(\mathrm{C}_{21}\right)$, 72ppm $\left(\mathrm{C}_{20}\right)$, 56ppm $\left(\mathrm{C}_{21}\right)$ , 161ppm( $\left.\mathrm{C}_{22}\right), 31 \mathrm{ppm}\left(\mathrm{C}_{23}\right), 164 \mathrm{ppm}\left(\mathrm{C}_{4}, \mathrm{C}_{7}\right), 196 \mathrm{ppm}\left(\mathrm{C}_{17}\right)$, 


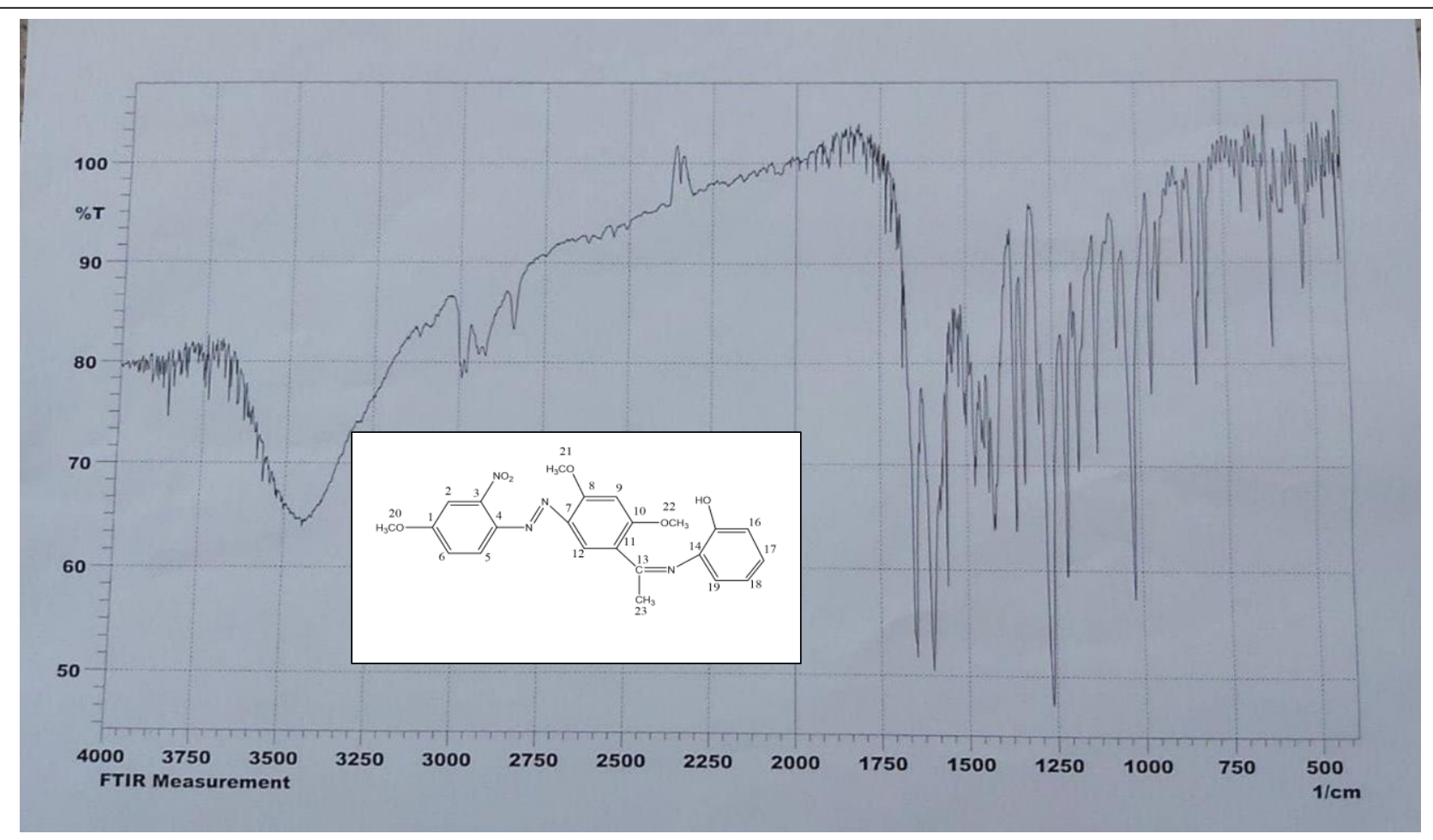

Fig(7) FT-.IR spectrum of.compound (B2) .

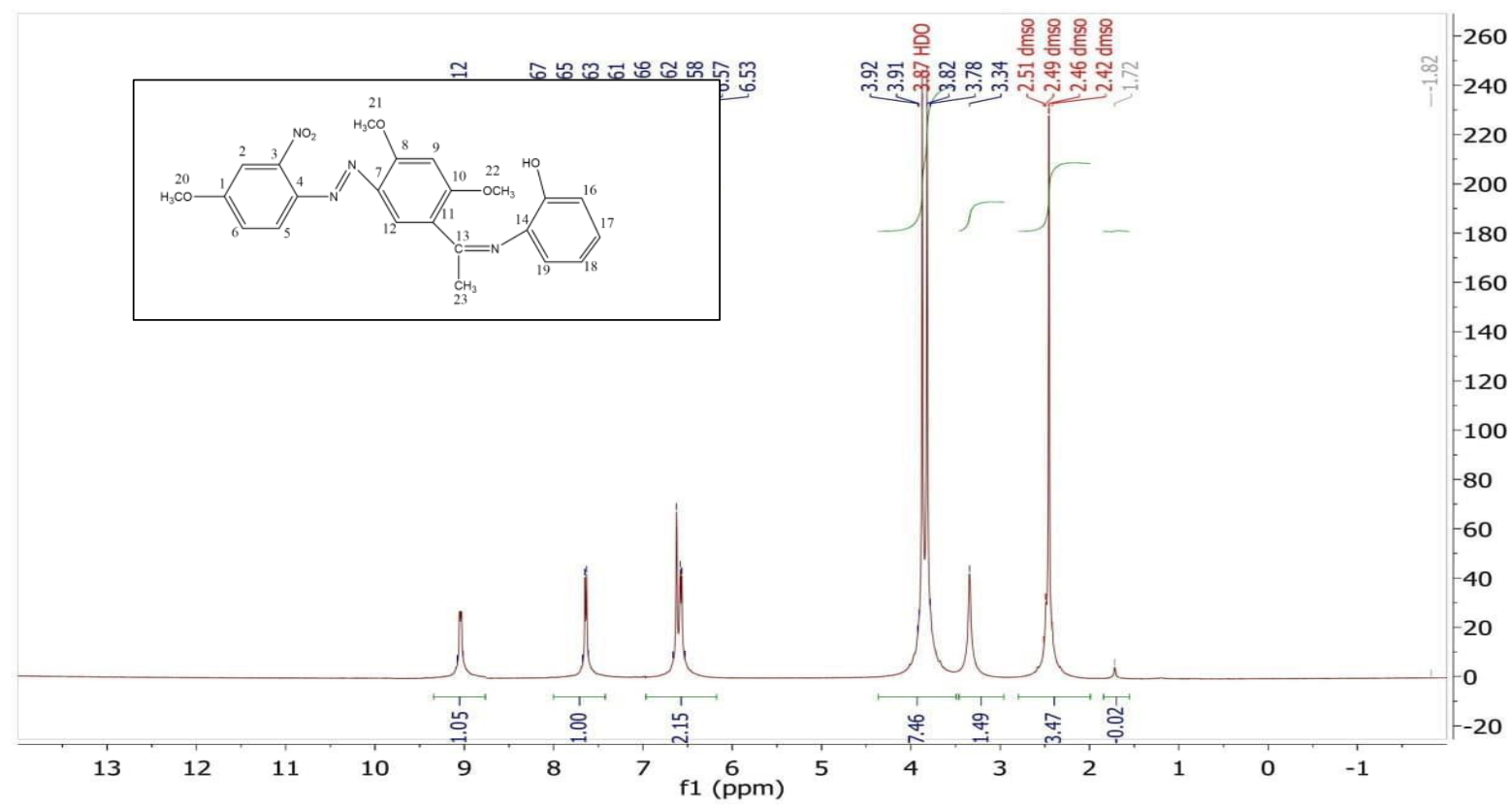

Fig(8) $\left({ }^{1}\right.$ H-NMR).spectrum, of.:compound (B2) 


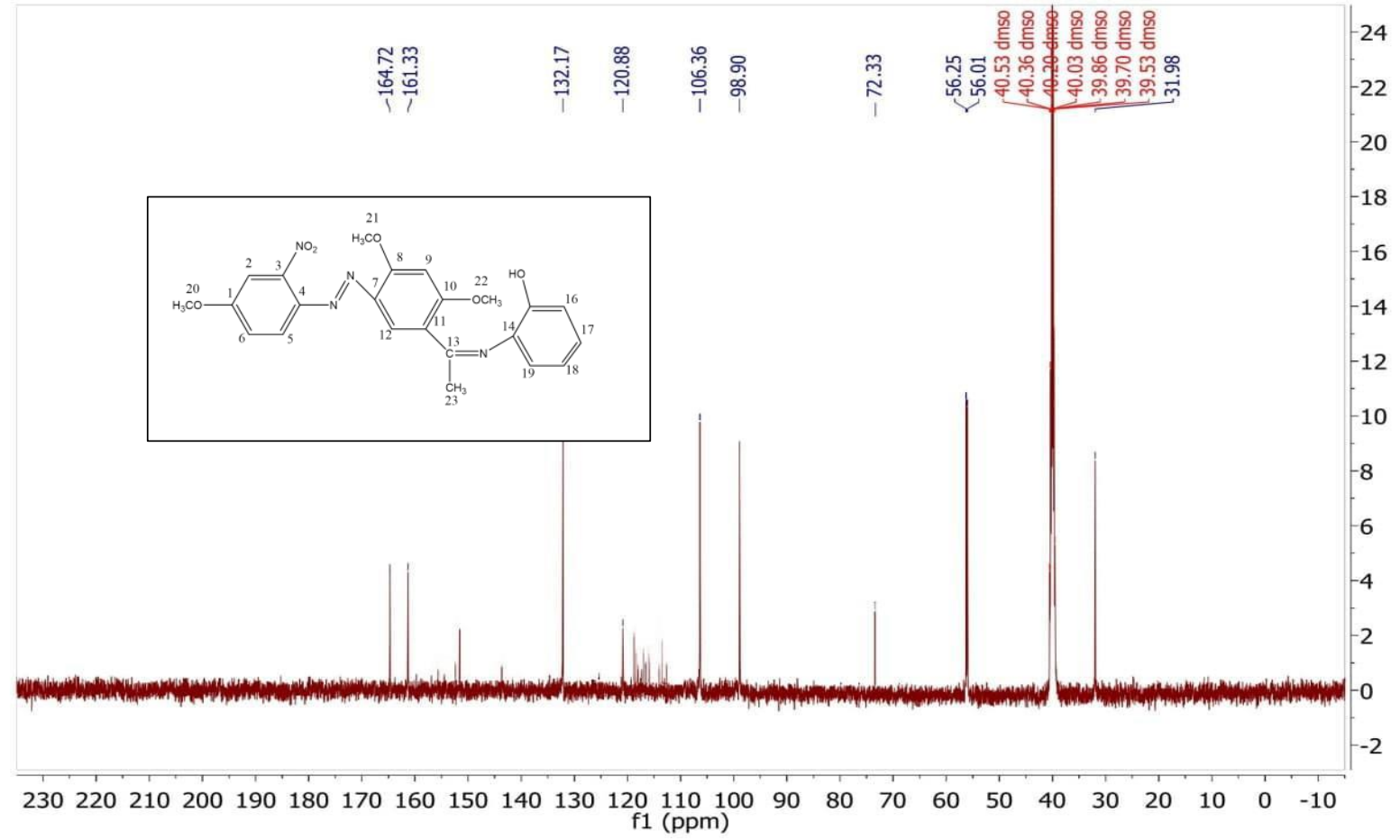

Fig,(9) ( ${ }^{13}$ C-NMR,) spectrum, of,compound (B2)

\section{Compound,(C1) $\quad 9$ ((E)-2-(2,6-dimethoxy-3-((4-methoxy-2-nitrophenyl)diazenyl)phenyl)-3-(4-} hydroxyphenyl)-2-methyl-2,3-dihydroquinazolin-4(1H)-one

FT-IR:,spectrum, data:,for derivative, (C1) show, peak at ,1420 for $(\mathrm{N}=\mathrm{N}), 3078$ for $(\mathrm{Ar}-\mathrm{H})$, $2947 \mathrm{~cm}-1$ for $(\mathrm{C}-\mathrm{H})$ in $\mathrm{CH} 3,1670 \mathrm{~cm}-1$ for $(\mathrm{C}=\mathrm{O})$, $1620 . \mathrm{cm}-1$ for $(\mathrm{C}=\mathrm{C}), 3425 \mathrm{~cm}-1 \mathrm{for}(\mathrm{N}-$ H).1HMNR.spectrum;, data, of derivative (C1) show 4.0ppm ( $\mathrm{s}, 1 \mathrm{H}, \mathrm{NH})$, 2.6.ppm $(\mathrm{S}, 3 \mathrm{H}, \mathrm{OCH} 3) 30$, 3.8.ppm ( $\mathrm{S}, 3 \mathrm{H}, \mathrm{OCH} 3) 28)$, 2.8.ppm $(\mathrm{S}, 3 \mathrm{H}, \mathrm{OCH} 3) 29,1.2 . p p m(\mathrm{~S}, 3 \mathrm{H}, \mathrm{OCH} 3) 27,(\mathrm{~s}, 1 \mathrm{H}, \mathrm{CH}), 1.8 \mathrm{ppm}(\mathrm{s}$, $2 \mathrm{H}, \mathrm{CH} 2), 6.5-7.8 \mathrm{ppm} 13 \mathrm{H} \quad,(\mathrm{Ar}-\mathrm{H}), 8.4 \mathrm{ppm} .(\mathrm{s}, 1 \mathrm{H}, \mathrm{OH}), 4.1 \mathrm{ppm} 9 \mathrm{~s}, 1 \mathrm{H} \mathrm{NH})$. C13_NMR spectrum data ( DMSO) compound (C1) show :79ppm (C27) ,56ppm(C28), 56.2ppm (C29) , 32ppm (C30), 31ppm (C19), 164ppm(C3 ),63ppm (C13) 196ppm(C14), 164-,98ppm (CArom) . 


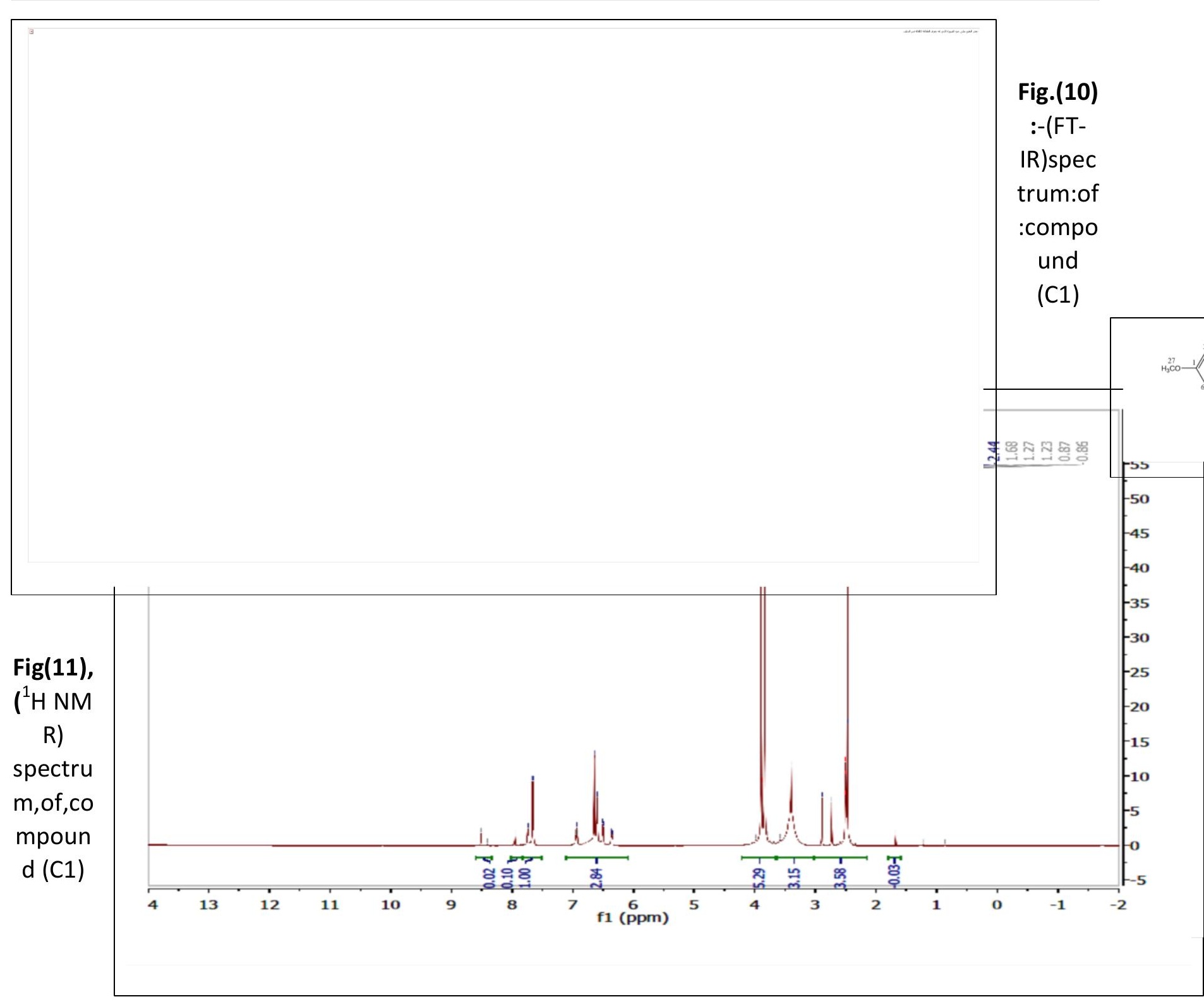




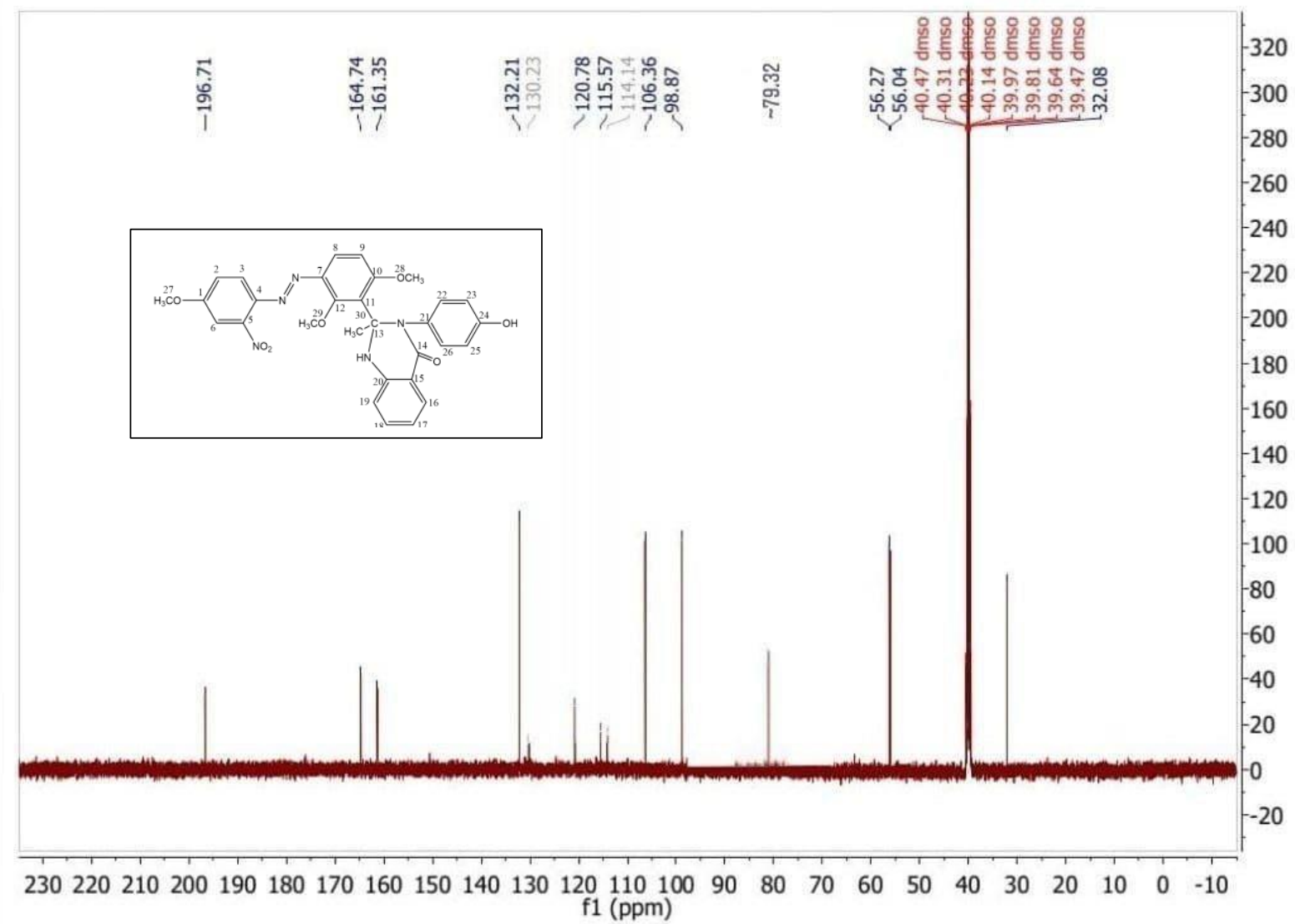

Fig(12):,- $\left({ }^{13}\right.$ C1-NMR), spectrum,.of.compound(C1)

Compound (C2 (E)-2-(2,6-dimethoxy-3-((4-methoxy-2-nitrophenyl)diazenyl)phenyl)-3-(2hydroxyphenyl)-2-methyl-2,3-dihydroquinazolin-4(1H)-one

FT-,.IR spectrum, :data for,derivative,(C2) show, peak at $3325 \mathrm{~cm}-1$ for, $(\mathrm{O}-\mathrm{H}$ ), , ,3060 for (Ar - H) , 2920cm-1 for (C- H) in CH3 , 1680, cm-1, for, (C=O) , 1600 cm-1, for (C=C),1550cm-1,(C=N),(15001360)cm-1NO2 . 1HMN, spectrum, data of,:compound (C2) show, ,2.8ppm(S,3H, OCH3)27) 2ppm (S ,3H, OCH3)26) ,3.9ppm ( $\mathrm{S}, 3 \mathrm{H}, \mathrm{OCH} 3) 25), 0.8 \mathrm{ppm}(\mathrm{s}, 3 \mathrm{H}, \mathrm{CH} 3) 29), 1.2 \mathrm{ppm}(\mathrm{s}, 3 \mathrm{H}, \mathrm{CH} 3) 28), 6.3-8.4 \mathrm{ppm}$ $(\mathrm{m}, 13 \mathrm{H}, \mathrm{Ar}-\mathrm{H}), .4 \mathrm{ppm}(\mathrm{s}, 1 \mathrm{H}, \mathrm{N}-\mathrm{H})$. The C13_NMR spectrum, data (DMSO). compound (C2) show :31ppm (C29), ， 32ppm (C28) , 56.05ppm (C27).,36ppm (C26),,56.28ppm(C25) 167ppm(C23),162,ppm(C21),164ppm,(C5-C7)),164-96 ppm(CArom),196(C=O). 


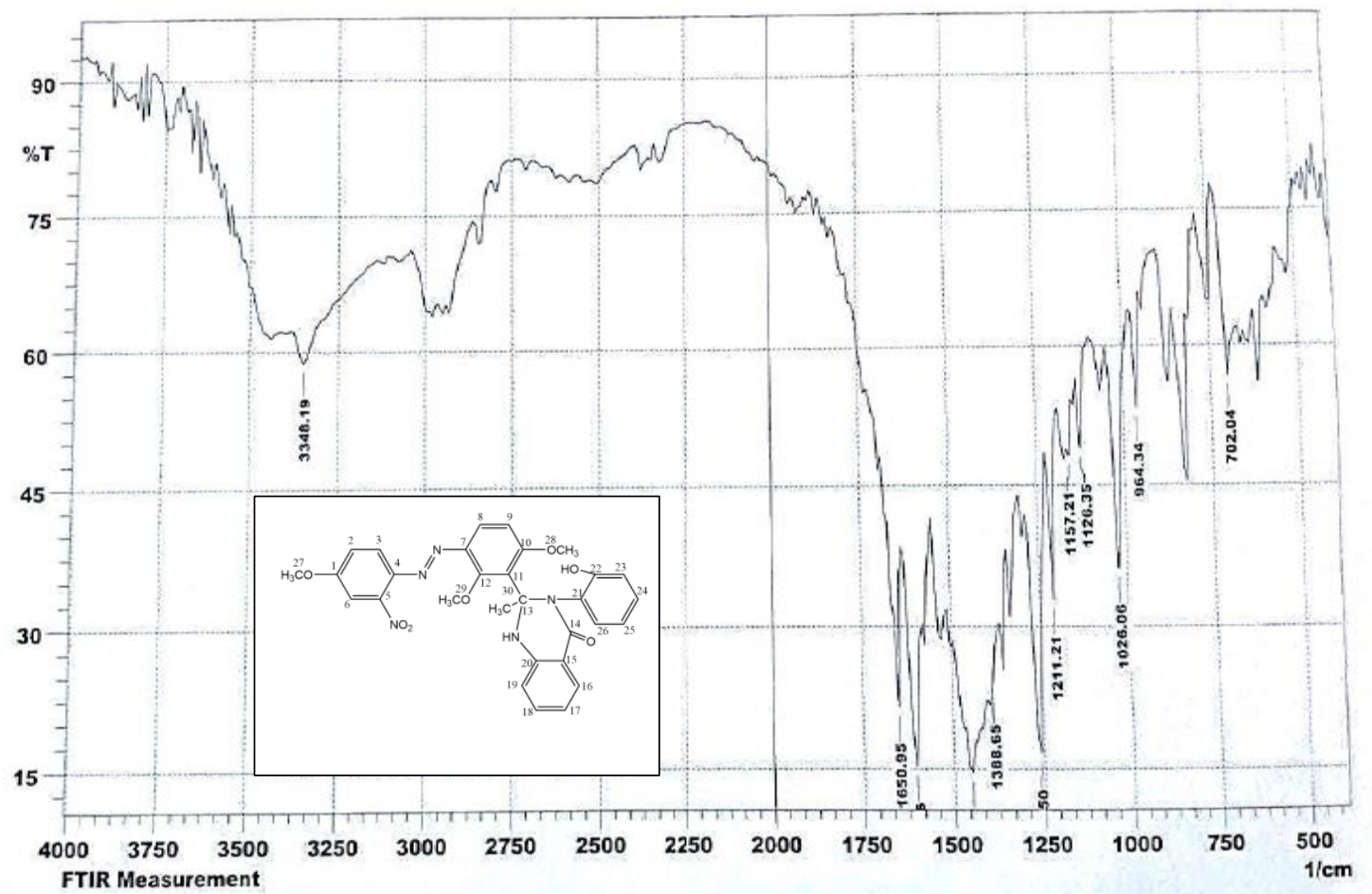

Fig(13):-( FT-IR)spectrum of:compound. (C2)

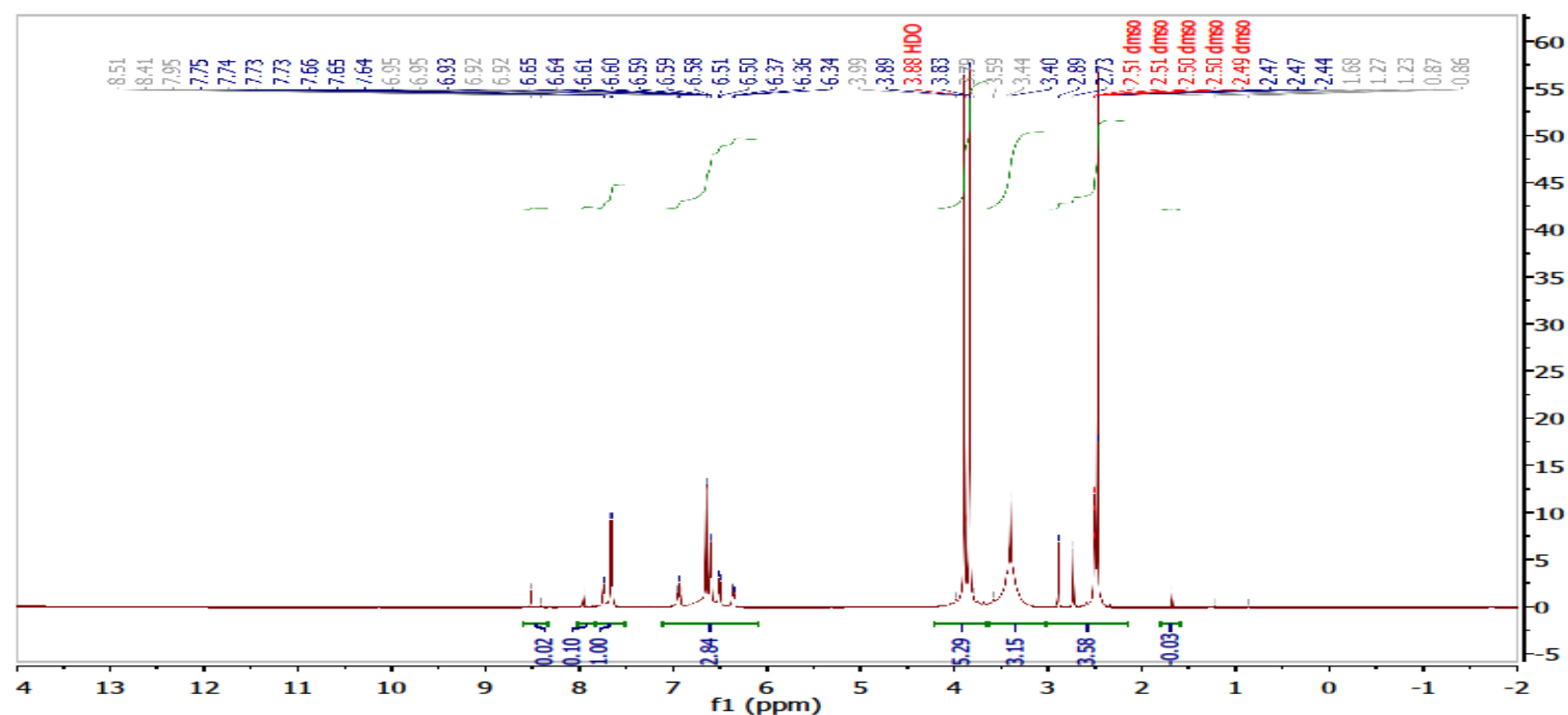

Fig(.14):-( ${ }^{1}$ H-NMR), spectrum, :of:compound (C2) 


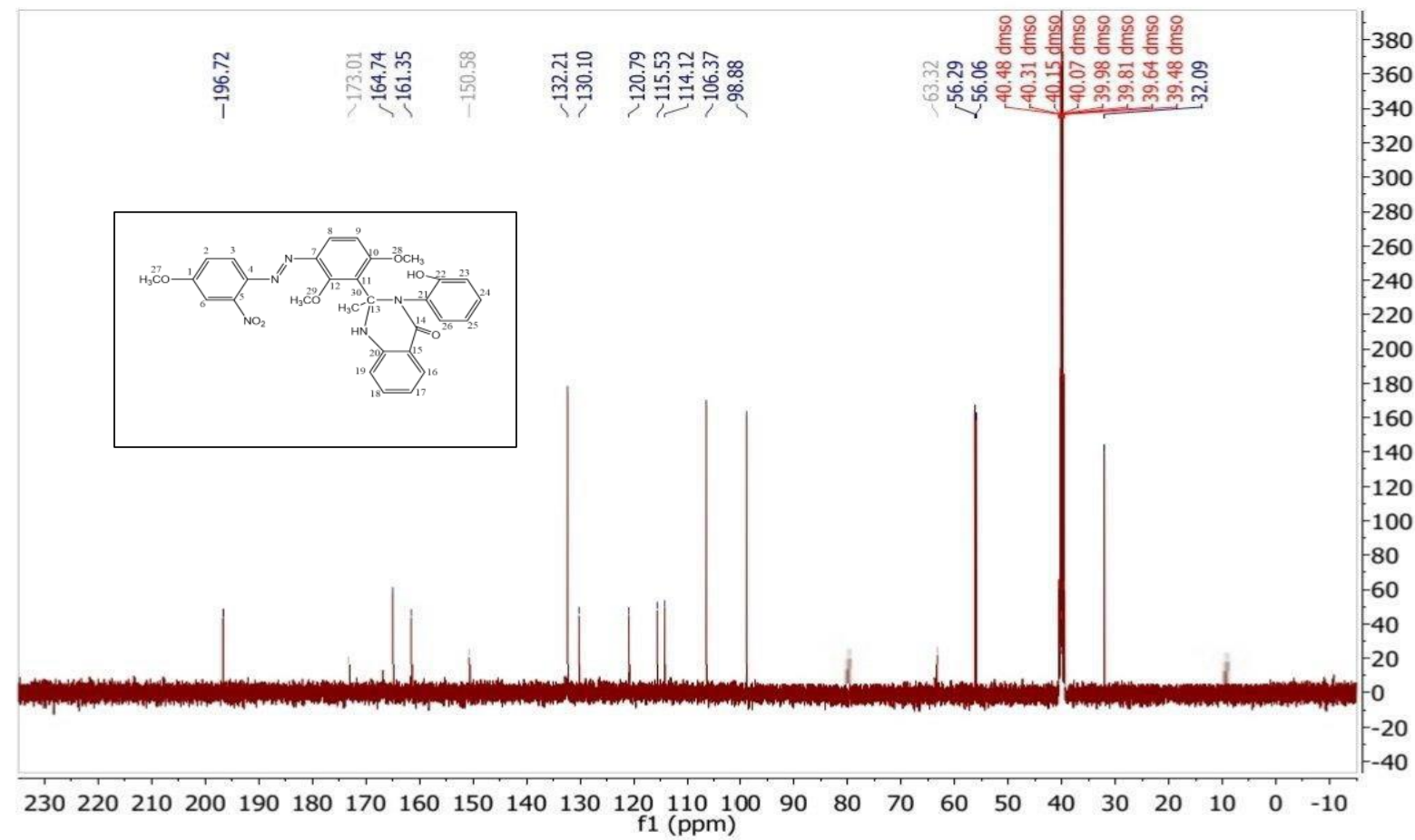

Fig:(15):- $\left({ }^{13} \mathrm{C} 1-\mathrm{NMR}\right)$ spectrum: of: compound (C2)

\section{Compound (D1):- $\quad$ ((E)-2-(2,6-dimethoxy-3-((4-methoxy-2-nitrophenyl)diazenyl)phenyl)-3-(4-} hydroxyphenyl)-2-methyl-2,3-dihydro-4H-benzo[e][1,3]thiazin-4-one

FT-IR spectrum:data for derivative (D1) show: peak at, 3090 for $(\mathrm{Ar}-\mathrm{H}), 2910 \mathrm{~cm}^{-1}$ for (C- H) in $\mathrm{CH}_{3}, 1680 \mathrm{~cm}^{-1}$ for $(\mathrm{C}=\mathrm{O}), 1412 \mathrm{~cm}^{-1}$ for $(\mathrm{N}=\mathrm{N}), 1600 \mathrm{~cm}^{-1}$ for $(\mathrm{C}=\mathrm{C}),(1550-1396) \mathrm{Cm}^{-1}$ for $(\mathrm{NO} 2) .{ }^{1} \mathrm{HMNR}$ spectrum:data of:compound (D1) show, 1.2ppm (S ,3H, OCH $\left.\left.)_{28}\right), 0.8 p p m\left(s, 3 \mathrm{H}, \mathrm{CH}_{3}\right)_{29}\right) 1.2 \mathrm{ppm}(\mathrm{S}$ $\left.\left., 3 \mathrm{H}, \mathrm{OCH}_{3}\right)_{28}\right)$, 3.8ppm (s , $\left.3 \mathrm{H}, \mathrm{OCH}_{(29)}, 2.4 \mathrm{ppm}\left(\mathrm{S}, 3 \mathrm{H}, \mathrm{OCH}_{3}\right)_{30}\right),(6.5-7.9) \mathrm{ppm}(\mathrm{s}, 13 \mathrm{H}, \mathrm{Ar}-\mathrm{H})$. The $\mathrm{C}^{13}$-NMR spectrum data ( DMSO) compound (D1) show :16.6ppm ( $C_{28}, 56 \mathrm{ppm}\left(\mathrm{C}_{30}\right), 153 \mathrm{ppm}$ $\left(C_{4}, C_{14}, C_{17}\right)$ 191ppm $\left(C_{31}\right), 111-134 p p m\left(C_{\text {Arom }}\right)$. 


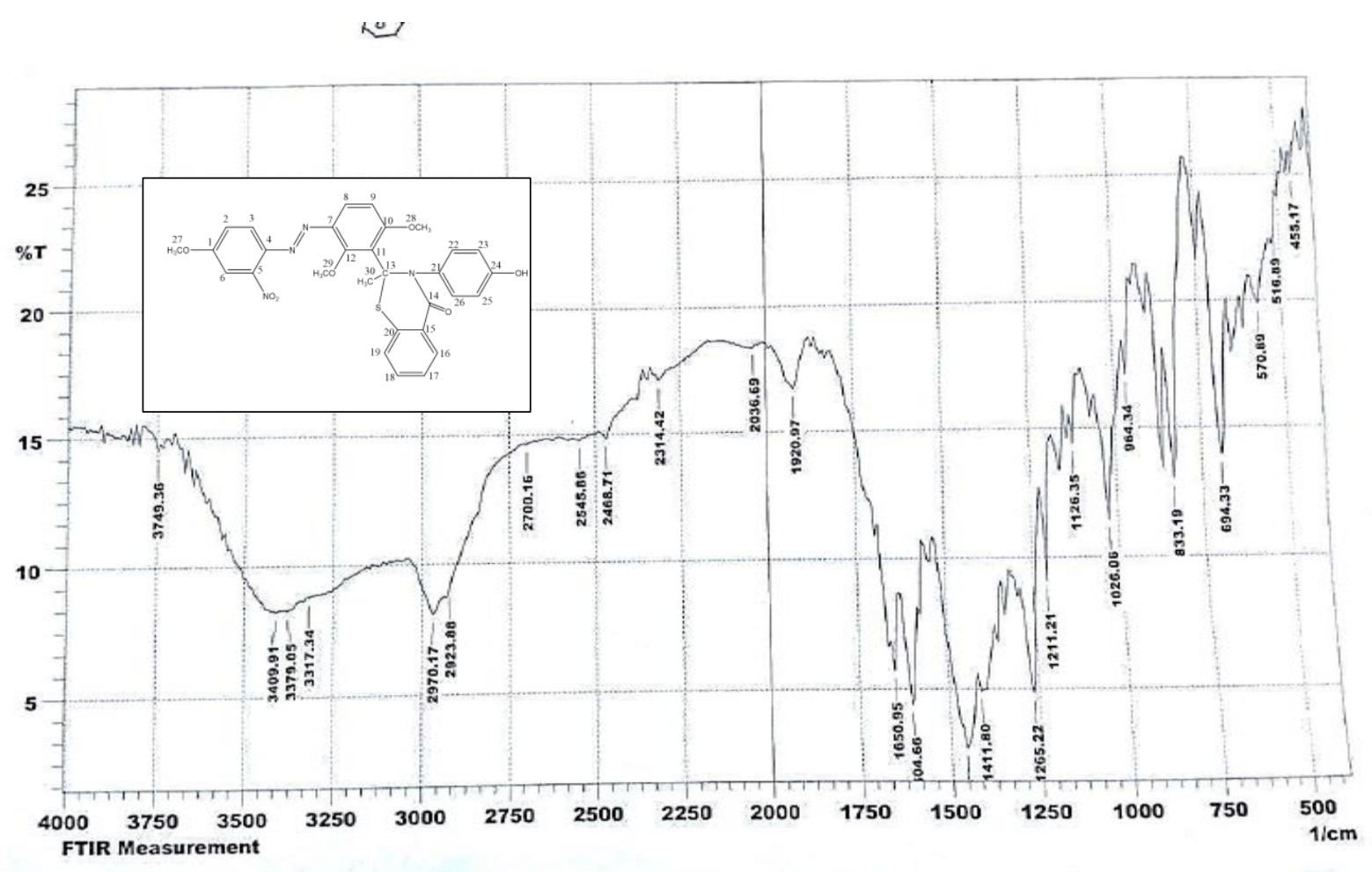

Fig(16):-( FT:-IR): spectrum of compound (D1)

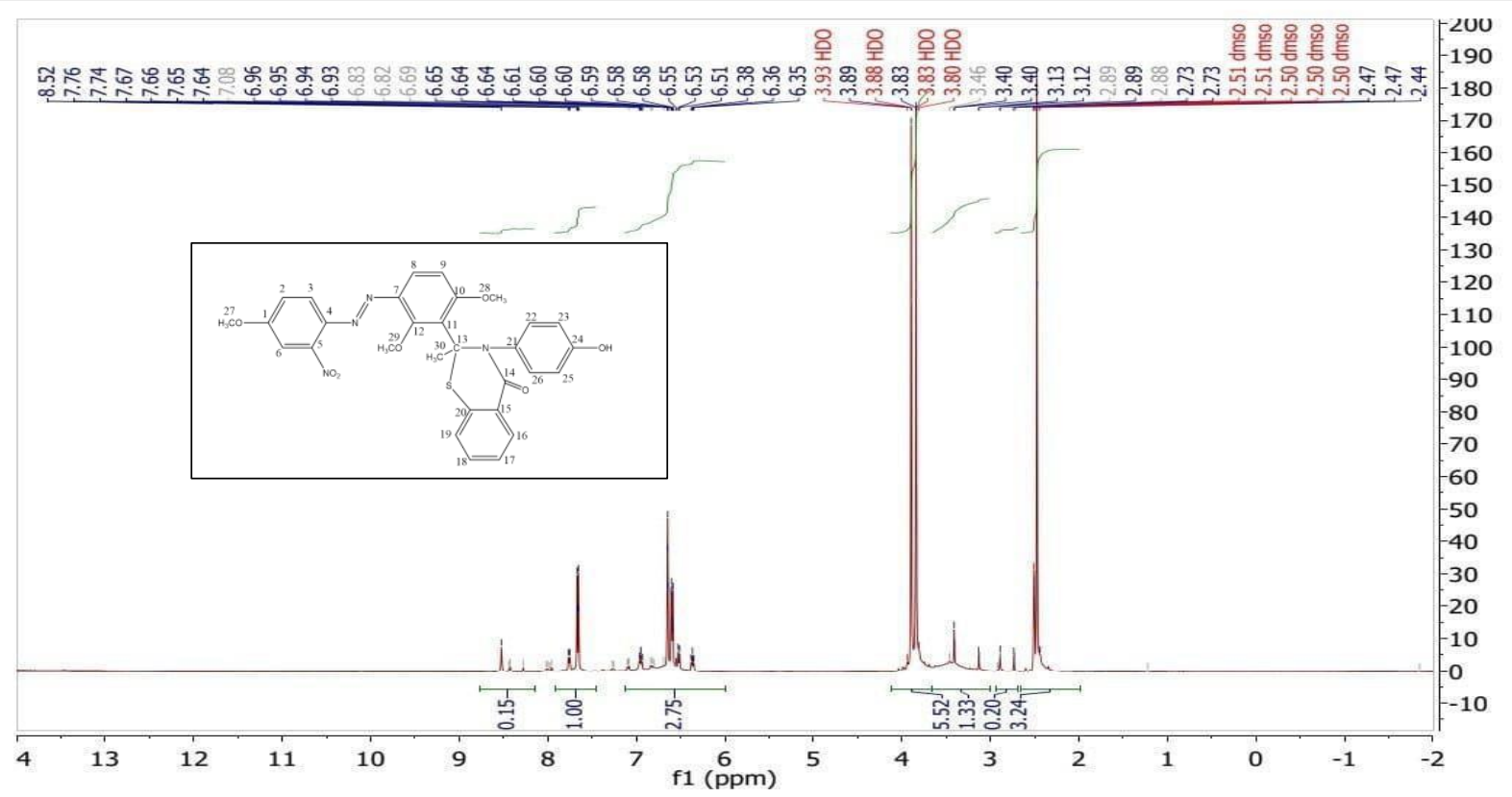

Fig(17):-H NMR, spectrum, of,compound (D1) 


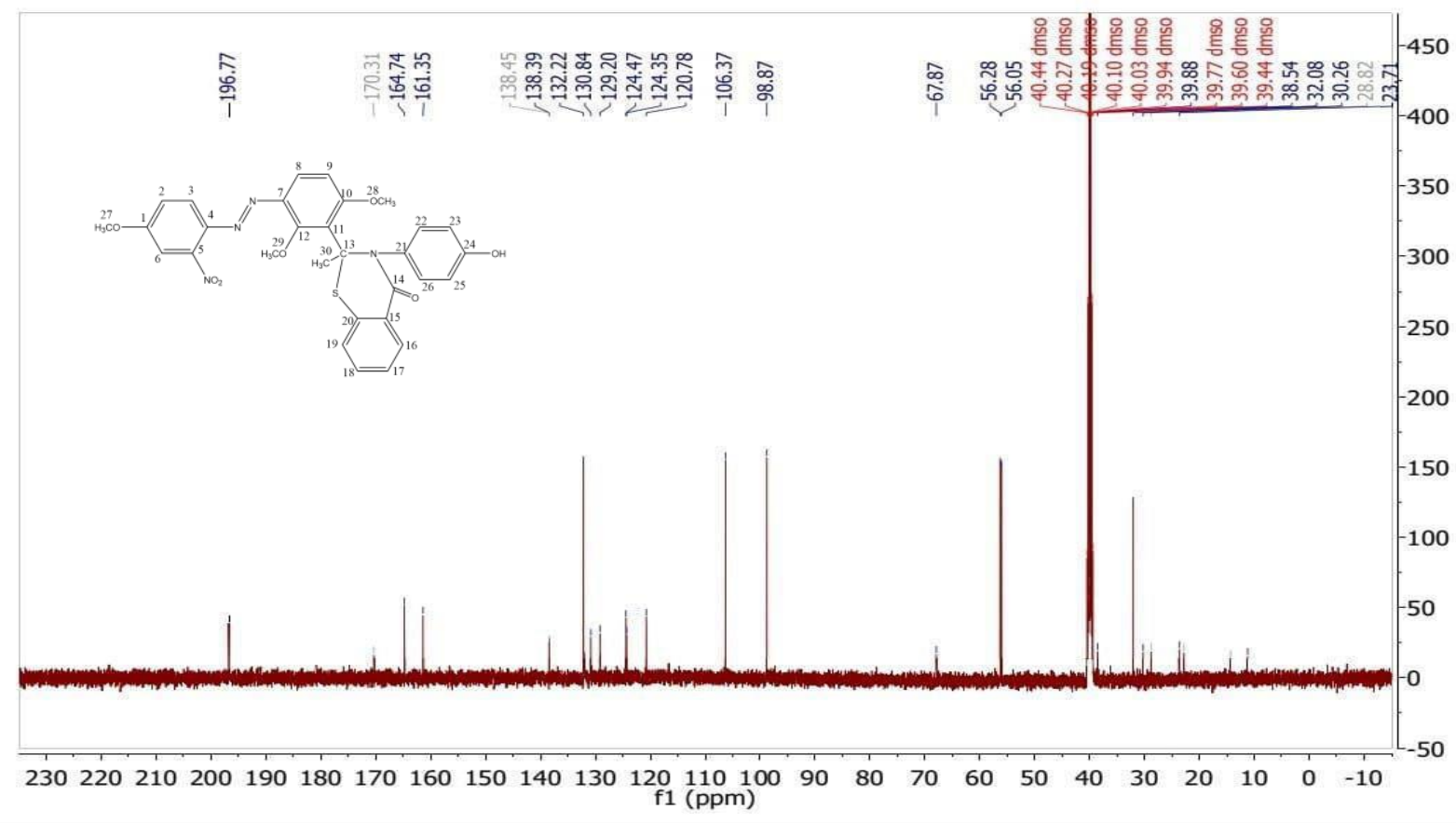

Fig(18):-( $\left.{ }^{13} \mathrm{C}-\mathrm{NMR}\right)$, spectrum, of compound (D1)

Compound (D2) (E)-,2-(2,6-dimethoxy-3,-((4-methoxy,-2-nitrophenyl)diazenyl)phenyl)-3-(5hydroxy-4-methylpyrimidin-2-yl)-2-methyl-:2,3-dihydro:-4H-benzo[:e][1,3]thiazin-4-:one

FT-IR:spectrum data for, derivative (D2), show:, band at $3332 \mathrm{~cm}^{-1,}$ for $(\mathrm{O}-\mathrm{H}),, 3080$ for , ((Ar $\mathrm{H}), 2931 \mathrm{~cm}^{-1}$ for $(\mathrm{C}-\mathrm{H})$ in $\mathrm{CH}_{3}, 1700 \mathrm{~cm}^{-1}$ for $(\mathrm{C}=\mathrm{O}),, 1458 \mathrm{~cm}^{-1}$ for $(\mathrm{N}=\mathrm{N}), 1600, \mathrm{~cm}^{-1}$ for $(\mathrm{C}=\mathrm{C})$ , $1650 \mathrm{~cm}^{-1}$ for $(\mathrm{C}=\mathrm{N}),(1535-1357) \mathrm{cm}^{-1}$ for $\left(\mathrm{NO}_{2}\right)$. ${ }^{1} \mathrm{HMNR}$ spectrum,data,of:compound (D2) show $\left.\left.\left.\left.0.8 \mathrm{ppm}\left(\mathrm{s}, 3 \mathrm{H}, \mathrm{CH}_{3}\right)_{28}\right), 1.2 \mathrm{ppm}\left(\mathrm{S}, 3 \mathrm{H}, \mathrm{OCH}_{3}\right)_{26}\right), 2.4 \mathrm{ppm}\left(\mathrm{s}, 3 \mathrm{H}, \mathrm{OCH}_{3}\right)_{27}\right), 3.8 \mathrm{ppm}\left(\mathrm{s}, 3 \mathrm{H}, \mathrm{OCH}_{3}\right)_{25}\right) 8.4$ ppm $(\mathrm{s}, 1 \mathrm{H}, \mathrm{OH}), 6.5-7.6 \mathrm{ppm}(\mathrm{m}, 10 \mathrm{H}, \mathrm{Ar}-\mathrm{H})$ The $\mathrm{C}^{13}-\mathrm{NMR}$ spectrum data ( DMSO) compound (D2) show :196ppm $\left(C_{14}\right)$, 36ppm $\left(C_{29}\right)$, 32ppm $\left(C_{28}\right), 32 p p m\left(C_{26}\right) 38 p p m\left(C_{27}\right), 56 p p m\left(C_{25}\right), 67$ ppm $\left(C_{13}\right)$ $30 p p m\left(C_{30}\right), 164 p p m\left(C_{4}, C_{7}\right)$. 


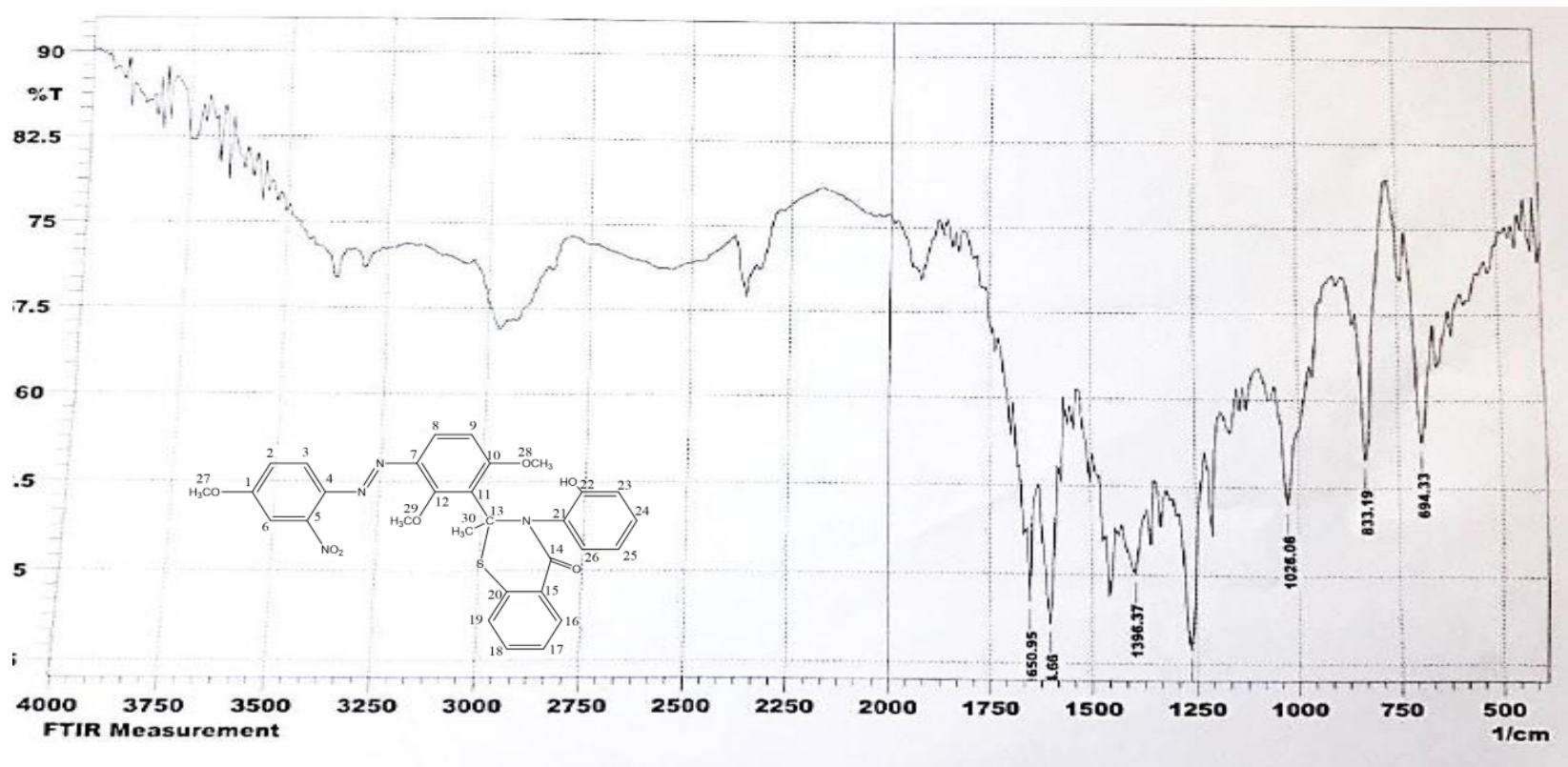

Fig(19):- (FT-IR) spectrum of: compound (D2)

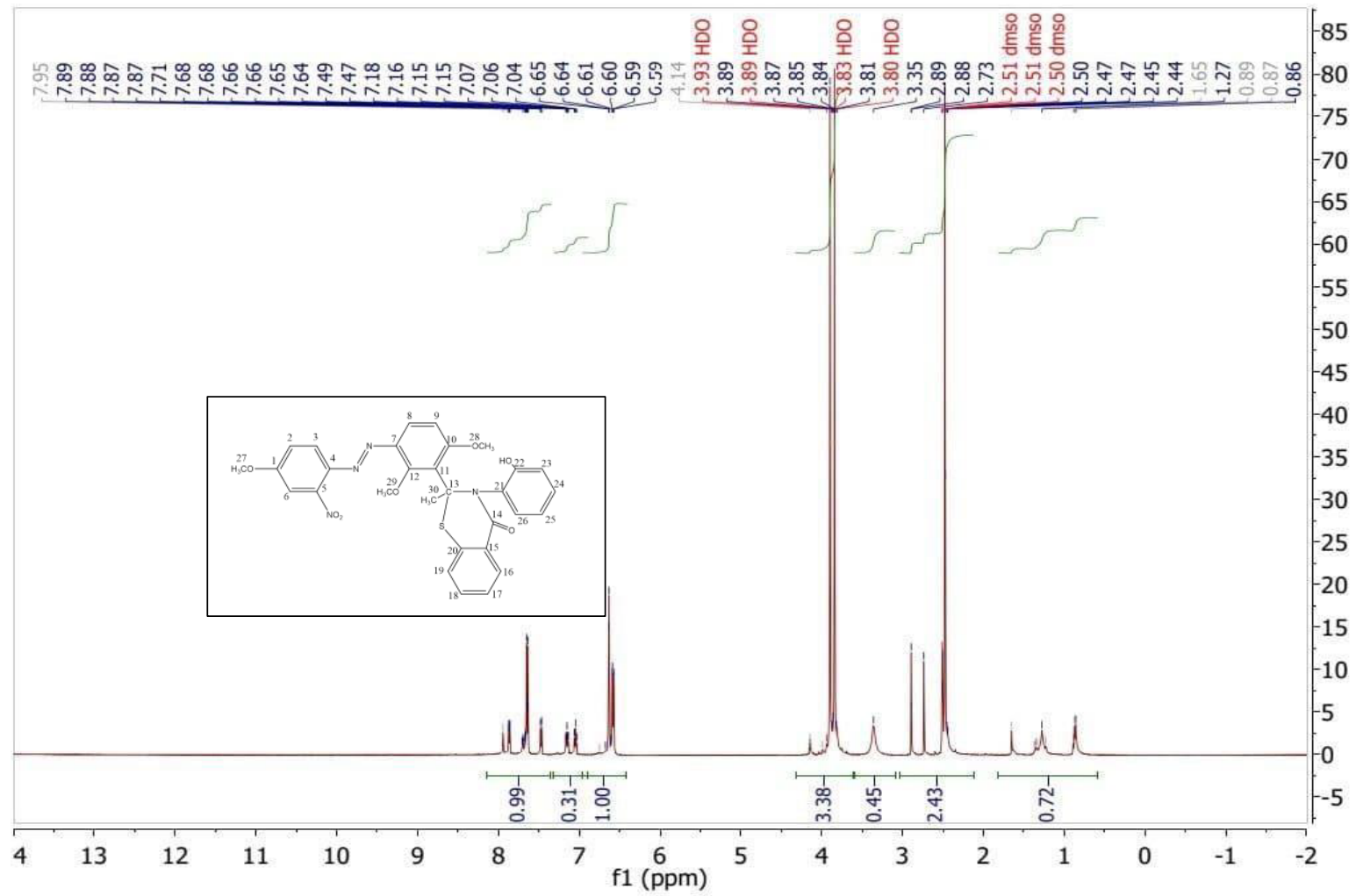

Fig(20):- $\left({ }^{1} H-N M R\right)$ spectrum ,of,compound (D2) 


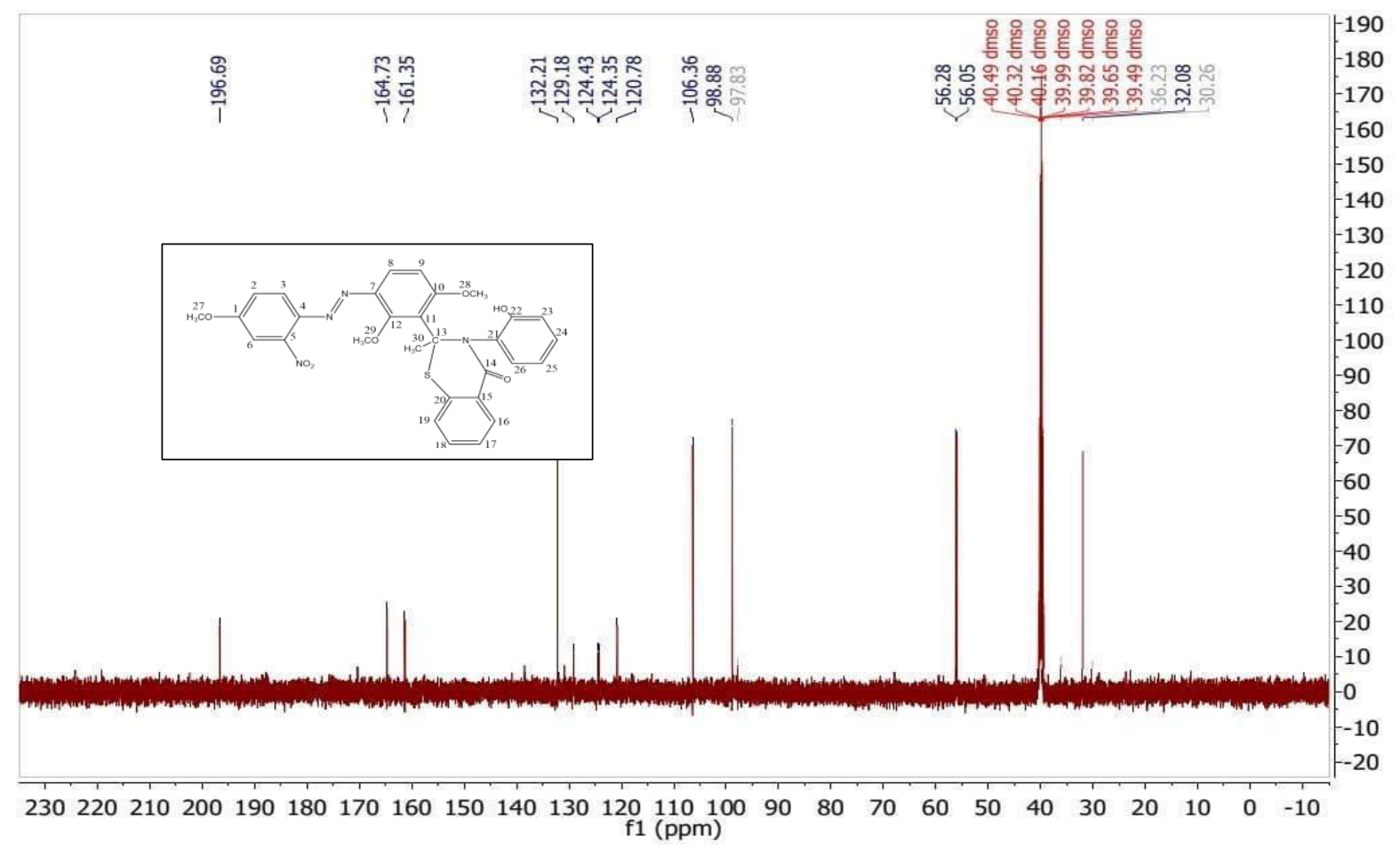

Fig(21):-( ${ }^{13}$ C-NMR:)spectrum:,of::compound (D2)

\section{Biological activity}

\section{1 antibacterial}

The results, show that,derivetives, reduce significant,antibacterial,effectiveness against bacteria "staphylococcus"aurous:and Escherichia:coli" . the compounds that show good:activity,are $(A, B 1, C 1, C 2$, ,D1) against (staphylococcus aurous), and compound that show very good activity are (A-D1),against.( Escherichia coli:,):,the results, of the antibacterial,activity are, shown:in,the fig (6). The results show that derivetives reduce significant antibacterial effectiveness against bacteria "staphylococcus aurous and Escherichia, coli", the compounds that show good activity are $(A, B 1, B 2, C 1, C 2, D 1, D 1)$ against (staphylococcus aurous) , and compound that show very good activity are (A-D1) against, ( Escherichia coli ) , the results of the antibacterial activity are shown in the fig (6). 


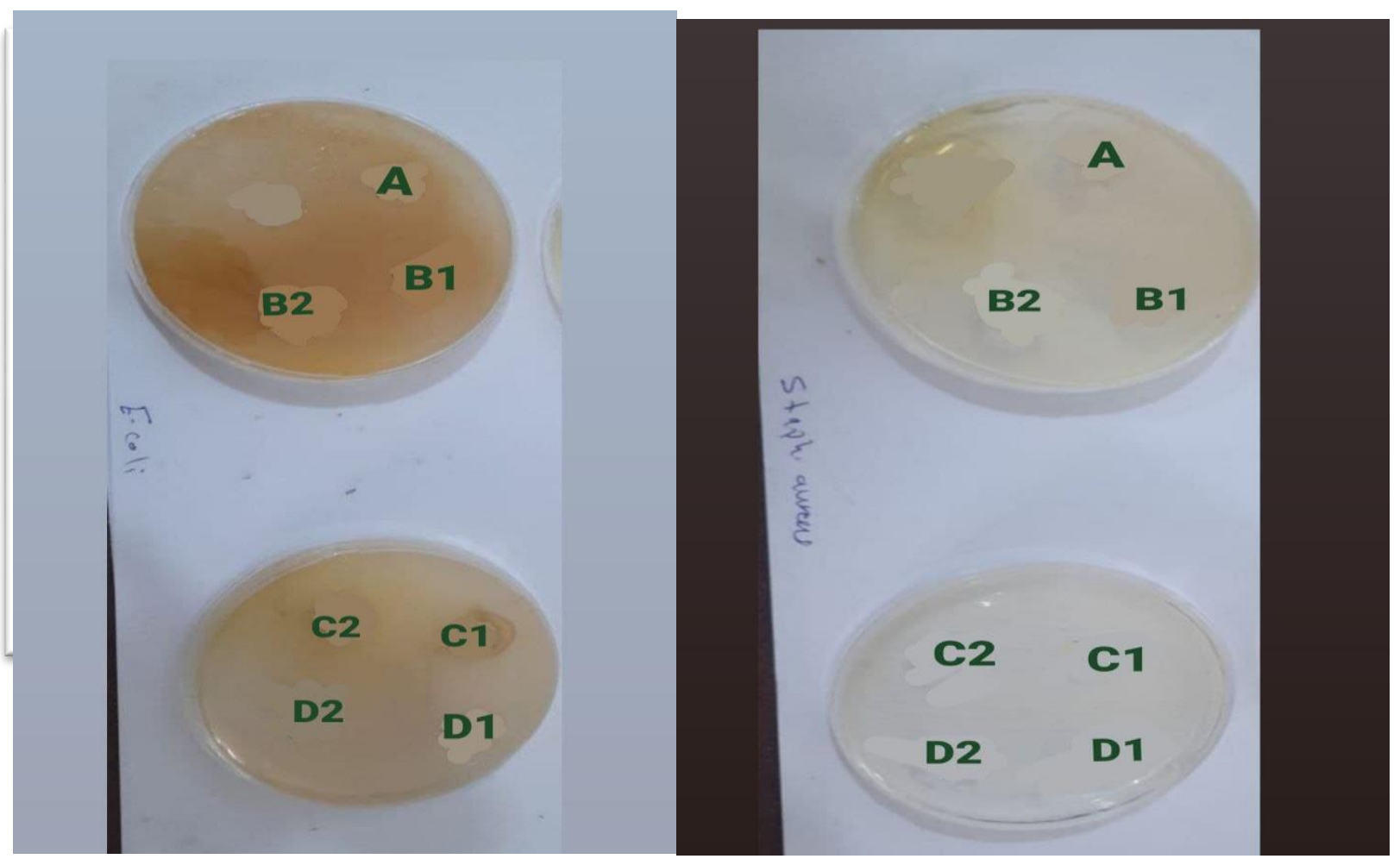

Fig, (30) effect: componds :(Staph Aureus) aginst and (E.Coli) aginst

Table (1) the results of the antibacterial activity for (A-D3) derivatives

\begin{tabular}{|c|c|c|c|c|}
\hline $\begin{array}{c}\text { Comp } \\
\text { No }\end{array}$ & Staph aureus & Mm & E.Coli & Mm \\
\hline A & +++ & 37 & +++ & 40 \\
\hline B1 & +++ & 45 & +++ & 30 \\
\hline B2 & - & 0 & - & Zero \\
\hline C1 & - & Zero & +++ & 45 \\
\hline C2 & - & Zero & - & Zero \\
\hline D1 & ++ & 22.3 & +++ & 30 \\
\hline D2 & +++ & 30.5 & - & Zero \\
\hline & & & & \\
\hline
\end{tabular}

\section{(-)No discouragementl}

"+= (5-10)mm , =slightly, :active, ++=, (11-20)mm moderately +++= More,than ,20: good, active" 


\begin{tabular}{|l|l|l|l|l|l|}
\hline Comp & M.F M.wat & m.p & $R f$ & Colour & $\%$ \\
\hline A & $\mathrm{C}_{17} \mathrm{H}_{17} \mathrm{~N}_{4} \mathrm{O}_{6} 359.11$ & 45 & 0.5 & Brown & 72 \\
\hline B1 & ${\mathrm{C} 23 \mathrm{H}_{22} \mathrm{~N}_{4} \mathrm{O}_{6} 450.5}$ & 120 & 0.47 & Black & 90 \\
\hline B2 & ${\mathrm{C} 23 \mathrm{H}_{22} \mathrm{~N}_{4} \mathrm{O}_{6} 450.5}$ & 115 & 0.5 & Brown & 93 \\
\hline C1 & $\mathrm{C}_{30} \mathrm{H}_{27} \mathrm{~N}_{5} \mathrm{O}_{7} 569.6$ & 180 & 0.4 & Black & 90 \\
\hline C2 & $\mathrm{C}_{30} \mathrm{H}_{27} \mathrm{~N}_{5} \mathrm{O}_{7} 569.6$ & 134 & 0.3 & Brown & 87 \\
\hline D1 & $\mathrm{C}_{30} \mathrm{H}_{25} \mathrm{~N}_{5} \mathrm{O}_{8} \mathrm{~S} 615.6$ & 160 & 0.2 & Black & 79 \\
\hline D2 & $\mathrm{C}_{29} \mathrm{H}_{26} \mathrm{~N}_{6} \mathrm{O}_{7} \mathrm{~S} 602.6$ & 182 & 0.29 & Black & 85 \\
\hline
\end{tabular}

\section{References}

1.Shrivastava, R., \& Gupta, P. Significant Antimicrobial Activity of some Schiff Bases. Page 4. Juni Khyat ISSN: 2278-4632 (2020) P.P . 276

2.. Kerru, N., Gummidi, L., Maddila, S., Gangu, K. K., \& Jonnalagadda, S. B. (2020). A review on recent advances in nitrogen-containing molecules and their biological applications. Molecules, 25(8), 1909.

3.Chulovska, Z., Chaban, T., Drapak, I.,: Matiychuk,:V., Chaban:I.,\& Nektegaev,I. Synthesis ofSome C5,Substituted 4-.Phenylimino.-Thiazolidin.-2-.Ones,as, Possible,Anti.Inflammatory,Agents. Biointerface, Research,in,Applied Chemistry, .. 11, . (2021). 8009.-8017.

4.Salman,S.:D., \&Adnan,:S..Synthesis, :characterization,of:Some $\quad$ \&ew.Derivatives of.(Oxazpine,Thiazinone and Hydroquinazoline),Azo,group,from,Amine,compounds.Eurasian,Chemico-Technological,Journal,20(3) . (2018). 264-276.

5.BDAIWI, Z. M., \& GHANEM, H. T. Synthesis,and,:Characterization: of, some heterocyclic,derivatives,from 2-amino thiazol,and study of,biological,activity of prepared derivatives. International Journal of Pharmaceutical Research, (2020),12(2).

6.Prakash,S.,: Somiya, G., Elavarasan, N., Subashini:, K.,Kanaga, S.,Dhandapani, R.,:\& Sujatha, V,Synthesis, and characterization, of novel,bioactive,azo,compounds 
fused, with:benzothiazole, and their,versatile,biological applications,. Journal of Molecular Structure, . 1224, (2018).,129016.

7.Balaji, G. L., Anusuya, V., \& Sujatha, G. Anti-oxidant, Anti,Inflammation,and,Antidiabetic Activity, of,Novel Schiff,Base of,Derived from,2-Hydroxy benzoic,acid (4-Chloro benzylidine).Hydrazide and.2-Hydroxy, benzoic acid (.3, 4-Dimethoxy, benzylidine)-Hydrazide.,European .Journal of,Molecular \&Clinical Medicine, 7(9,(2020.). 1760-1769.

8. Anusuya, V., Sujatha, G., Broheshnu, G. B., \& Balaji, G. L. (2020). Anti,oxidant,.Anti.Inflammation, and Anti,Diabetic,Activity of,Novel Schiff.Base,Derived,from,2Hydroxy,benzoic,acid (.3-Hydroxy benzylidine);-Hydrazide and 2-Hydroxy benzoic acid (3-Hydroxy-4methoxy benzylidine)-Hydrazide.European, Journal.of,Molecular .,Clinical.Medicine,,7(4),. 2523-2532.

9.Fabbrizzi,L.Beauty, in,Chemistry: Making.Artistic,Molecules, with Schiff,Bases.The. Journal,of Organic Chemistr.y, 85(19), (2020).12212-12226.

10.Liu, H. Long, S.,Rakesh. K. P., \&,Zha,.G.F.Structure.-activity.relationships (SAR)of .triazine,derivatives: Promising,antimicrobial,agents..European journal,of medicinal,chemistry „,185, . (2020). 111804.

11.Hadi, M. A., \& Kareem, I. K. (2020). Synthesis,Characterization.and Spectral,Studies of a New AzoSchiff base Ligand. Derived,from 3, 4-diamino benzophenone and its Complexes with Selected Metal Ions. Research Journal in Advanced Sciences, . (2020). 1(1).

12.IBRAHIM, H. A., AL-MAJIDI, S. M., \& AL-ISSA, Y. A. Synthesis,and identification.of some, new N.substituted quinazoline-.4-one, thiazine-4-.one and,tetrazoline rings,incorporating.N-ethyl-2(benzylthio),benzimidazole acetate,and:study,their,application,as:anti-oxidant,agent.,International Journal,of.Pharmaceutical.Research, (2020). 12(3).

13.Ghodge,. B.,:Kshirsagar. A., \&:Navghare,. V.:Synthesis,.characterization,.and,iinvestigation.of the, anti-inflammatory, effect, of $\quad 2, \quad 3$-disubstituted,quinazoline-4.(1H)-one.,Beni-Suef, University Journal of Basic and Applied Sciences, 9(1) (2020)., 1-12. 
14.Liu., H., Long., S.,:Rakesh,. K. P., \& Zha, G..: F. Structure-.activity relationships:(SAR) of:triazine derivatives: Promising.antimicrobial.agents. European,journal,of medicinal,chemistry,185, (2020). 111804.

15. Sherif, S. H., Kure, D. A., Moges, E. A., \& Argaw, B. (2021). Synthesis., Characterization, and Antibacterial,Activity,Evaluation of 4-amino Antipyrine.Derivatives and Their Transition Metal Complexes. American Journal of Bioscience and Bioengineering, 9(1), 8-12.

16. Yousif, E. I., Hussien, A. K., \& Hasan, H. A. (2020). COII, NIII AND CDII COMPLEXES. DERIVED .FROM,MIXED:,AZO-LINKED,SCHIFF-BASE,LIGANDS:.FORMATION,,CHARACTERISATION, THERMAL:,ANALYSIS ,AND :BIOLOGICAL,,STUDY, Plant,Archives, 20(1), 2405-2411.

17.Kareem, A. F., \& :Ghanim, H. T:. Antimicrobial Study against Seven Cycles Compounds Derivatives from Pyrimidine. Indian. Journal of .Forensic,Medicine \&,Toxicology, 14(3), (2020). 1515.

18.. Kaur, R., \& Kumar, K. (2021). Synthetic,and,medicinal..perspective.of,quinolones.as,antiviral agents.European:,Journal of,Medicinal.Chemistry, 113220.

19. Fonkui,T.Y., Ikhile, M..I., Muganza, F.. M., Fotsing,. M. C.. D., Arderne, C., \& Siwe-Noundou, X. (2018). Synthesis,:characterization and biological,applications,of, novel,Schiff,bases,of.2.(trifluoromethoxy) aniline. 中国药学 (英文版), 27(5), 307-323.

20. Das, R., Mehta, D. K., :\& Dhanawat,: M. (2020). Bestowal of Quinazoline Scaffold in Anticancer Drug Discovery.Anti.-Cancer,Agents in.Medicinal.Chemistry,.20, 00-00.

21.BDAIWI, Z. M., \& GHANEM, H. T. Synthesis.and,Characterization..of some.heterocyclic.derivatives from .2-amino. thiazol and study. of biological,activity of prepared derivatives. International Journal of Pharmaceutical Research, (2020). 12(2).

22.Behjet, S. A., \& Khudair, Z. F.: (Synthesis,and:Characterization. Of.Some New. Heterocyclic Derivatives and Studying, of their,Biological Activity (Anti-Bacteria). INTERNATIONAL JOURNAL OF PHARMACEUTICAL AND CLINICAL RESEARCH, 11(01), (2020). 38-44. 